\title{
FINITE EIGENFUNCTION APPROXIMATIONS FOR CONTINUOUS SPECTRUM OPERATORS
}

ROBERT M. KAUFFMAN

\author{
Department of Mathematics \\ University of Alabama at Birmingham \\ Birmingham Alabama 35294
}

(Received January 23, 1992)

\begin{abstract}
In this paper, we introduce a new formulation of the theory of continuous spectrum eigenfunction expansions for self-adjoint operators and analyze the question of when operators may be approximated in an operator norm by finite sums of multiples of eigenprojections of multiplicity one. The theory is designed for application to ordinary and partial differential equations; relationships between the abstract theory and differential equations are worked out in the paper. One motivation for the study is the question of whether these expansions are susceptible to computation on a computer, as is known to be the case for many examples in the discrete spectrum case. The point of the paper is that continuous and discrete spectrum eigenfunction expansions are treated by the same formalism; both are limits in an operator norm of finite sums.
\end{abstract}

KEY WORDS AND PHRASES. Continuous spectrum eigenfunction expansion, self-adjoint operator, ordinary differential operator, partial differential operator, spectral theorem.

1991 AMS SUBJECT CLASSIFICATION CODES. Primary 47A70, 47E05, 34L10, 35P10.

\section{INTRODUCTION}

Eigenfunction expansions may be considered as an abstraction of the idea of approximating complicated waves by finitely many standing waves. For discrete spectrum eigenfunction expansions associated with a self-adjoint operator $H$ in a Hilbert space $\mathrm{L}_{2}(\mathrm{X}, \rho)$, the rate of convergence of the expansion has been the subject of a great deal of research. In the continuous spectrum case, sums are replaced by integrals, and the question of whether the integral can be approximated by a finite sum has not been studied. This paper begins such a study; first, however, we indicate why the question is important, and what sort of answers we look for. It is helpful to take a naive look at the method of separation of variables, or eigenfunction expansions.

One purpose of an eigenfunction expansion is to convert continuous data, such as functions, into elements of $\mathbb{C}^{\mathrm{n}}$, vectors formed from the coefficients of the function in the expansion. A problem, such as a partial differential equation, in a function space is then transferred to $\mathbb{C}^{\mathbf{n}}$, solved there (partial differential equations often transform into ordinary differential equations with constant coefficients), and then the solution to the original problem is obtained by 
transforming back, that is, by summing the eigenfunctions with the recalculated coefficients. This involves a certain error. The error is measured using two Hilbert spaces $\mathrm{Y}$ and $\mathrm{Z}$.

An eigenprojection in $B(Y, Z)$, the bounded operators from $Y$ into $Z$, of a self-adjoint operator $\mathrm{H}$ in a Hilbert space $\mathrm{L}_{2}$ is an operator in $\mathrm{B}(\mathrm{Y}, \mathrm{Z})$ of the form $\mathrm{P}(\phi)=\mathrm{F}(\phi) \mathrm{F}$, where $F \in Z \subset Y^{\prime}$, and where $H$ takes a dense locally convex vector space $W$ contained in $Y$ continuously into itself, and $H^{\prime} F=\lambda F$. Ideally, the operator which solves the original problem is the limit in $B(Y, Z)$ of finite sums of multiples of eigenprojections. We call this property the discrete approximation property, for the remainder of this introduction.

If the discrete approximation property holds, the eigenfunctions used to perform the expansion do not have to be recalculated for each new function being expanded, and only finitely many coefficients must be calculated. In other words, up to a certain error, functions become elements of $\mathbf{C}^{\mathrm{n}}$, and semigroups generated by the original self-adjoint operator become semigroups of diagonal matrices. Continuous spectrum expansions have at present no such theory; these are modelled on the inverse Fourier transform, so instead of finite sums one must work with integrals which in general only converge in the mean, and convergence in operator norm is not discussed. One consequence of this is that the theory of continuous spectrum eigenfunction expansions appears to have no computational significance; it seemingly cannot be put onto a computer. This situation, which if true would lead to problems of whether the theory is well-posed in any reasonable sense, contradicts the intuition gained from the Fourier transform; the Fourier transform is well-known to be computationally significant. The purpose of this paper is to begin a continuous spectrum theory modelled on the discrete spectrum case, where finite sums appear instead of integrals. In order to do this, it has been useful to reformulate the existing theory of these expansions. More about this reformulation will be given later in this introduction.

The discrete approximation property is shown in section 3 to follow if the operator in question is a convergent operator-valued integral, in $B(Y, Z)$, of eigenprojections, which in the continuous spectrum case must go from a space $Y$ smaller than $L_{2}$ to a space $Z$ large enough to contain the eigenfunctions. Hence we must study when the expansion is such an integral. This is shown in section 3 to be true when the measure of $\Delta$ is finite, with respect to an invariant measure depending for a cyclic subspace only upon $\mathrm{Z}$. This measure is the one which normalizes the eigenfunctions in $\mathbf{Z}$.

For Sturm-Liouville theory for the Dirichlet problem on a finite interval, with $\mathrm{Y}=\mathrm{H}_{0}^{1}$ and $\mathrm{Z}=\mathrm{L}^{\infty}$, the sort of convergence we study is well known to occur, as it does in many other discrete spectrum problems. Even in discrete spectrum problems, however, the calculation of appropriate spaces $\mathrm{Y}$ and $\mathrm{Z}$ is often nontrivial. In this paper, in the discrete or continuous spectrum case, they are calculated using a priori estimates on the domain of $H$. Eigenfunctions satisfy the equation $\mathrm{H}^{\prime} \mathrm{F}=\lambda \mathrm{F}$ in a certain dual space and are members of $\mathrm{Z}$.

To study the discrete approximation property for an arbitrary bounded continuous function of $H$, we show that it is sufficient to study the property for the spectral projections $P(\Delta)$ for $H$, corresponding to Borel sets $\Delta$. We show in section 5 that the discrete approximation property for $\mathrm{P}(\Delta)$ is equivalent to the question of whether $\mathrm{P}(\Delta)$ is compact in $\mathrm{B}\left(\mathrm{Y}, \mathrm{L}_{2}\right)$, a question of interest in its own right. We obtain results which give compactness for concrete examples in spaces where it is otherwise not known. This shows that the theory has consequences which reach beyond itself, and suggests the problem of characterizing the sets $\Delta$ such that $\mathrm{P}(\Delta)$ is compact; for examples such as the time-dependent Schrödinger equation, this means calculating the energy sets on which 
the separation of variables scheme discussed earlier works.

It is not quite accurate to say that existing theories completely ignore these questions. A little bit of thought will convince one that for a bounded set $\Delta$, the inverse Fourier transform representation of the spectral projection $\mathrm{P}(\Delta)$ for the self-adjoint operator associated with id/dx has discrete approximation properties from, say, $\mathrm{Y}=\left\{\theta: \theta / \omega \in \mathrm{L}_{2}\right\}$ to $\mathrm{Z}=\left\{\mathrm{F}: \omega \mathrm{F} \in \mathrm{L}_{2}\right\}$, where $\omega$ is a well-behaved $\mathrm{L}_{2}$ function. From the equivalence, shown in section 5 , of the approximation property for $\mathrm{P}(\Delta)$ with the compactness of $\mathrm{P}(\Delta)$ in $\mathrm{B}(\mathrm{Y}, \mathrm{Z})$, together with known results about Schrödinger operators, it is not difficult to establish the same properties for the Schrödinger operator with a well-behaved potential, where $\Delta$ is a bounded set of energies. However, in situations like this, the compactness of the spectral projection is known beforehand, and may be used as in Section 5 to produce the approximation property. A more interesting problem is that of of unbounded sets $\Delta$. With the $Y$ and $Z$ above, for the inverse Fourier transform, it turns out that some unbounded sets $\Delta$ have this property and others do not. The compactness of $P(\Delta)$ is only known as a consequence of the theory. The results of this paper are oriented toward the study of which sets $\Delta$ have this property. As an example, the general Sturm-Liouville case on a half-line is studied in section 4. Much sharper results for short-range potentials are given by $D$. B. Hinton and the author in [4], using the results of this paper. Examples from partial differential equations also fit easily into the formalization of this paper and explicit examples are given. Here the results are less sharp unless one restricts to a single cyclic subspace.

The heart of the paper is the operator valued integrals of Section 3, together with their relation to the approximation problem. In section 5 , we show the equivalence of the two problems, compactness and approximation. Using the results of sections 3,4 and 5 of the paper, we see that certain spectral projections $\mathrm{P}(\Delta)$ are compact as operators between spaces where they are not already known to be compact by a priori estimates. A simple example is given in section 4 , which is about second order ordinary differential operators.

The theory of continuous spectrum eigenfunction expansions is a very old one, going back to Gelfand's work in the 1950's. The book of Berezanskii [1] is a fundamental reference, but it is difficult to extract specific information from such a general theory. The work of Simon [8], which is functional-analytic though it is specifically slanted toward Schrödinger operators, is a clear and rigorous approach to the theory with a lot of specific information. The paper of Poerschke-Stolz-Weidmann [5] is more general, and also has more elementary proofs. This paper has been followed up by Poershke and Stolz [6], who give applications of their results to scattering theory.

With such a large and excellent literature, why give yet another approach to the whole theory? We do so, partly to obtain the crucial assertion iii) of Lemma 1.6, which we need for the basic problems discussed earlier, but also to be able to analyze the expansion in a format based simply upon a priori estimates on the domain of powers of the self-adjoint operator $\mathrm{H}$ which is being decomposed, so as to make the results as concrete as possible for applications to differential equations. The question of what is needed about an a priori estimate in order to do this is answered in the paper. Our proofs are self-contained, since once the formalism is set up and Lemma 1.6 is proved, the inverse Fourier transform (Lemma 3.4) and the Fourier transform (Theorem 1.8) follow quite directly from the spectral theorem; to attempt to invoke other results would introduce technical difficulties. The estimates on the eigenfunctions in this paper contained in Assertion ii) of Theorem 1.8 do not follow (at least directly) from other results; and as was remarked earlier the kind of strong convergence of the integrals in the inverse transform contained 
in Lemma 3.4 is not studied at all in existing literature. On the other hand, our hypotheses are different from those of other approaches such as [5] and [8]; for example, our theory also demands more smoothness on the coefficients when applied to differential equations, as we discuss below. The relationship between this work and that of [5], [6] and [8] is an interesting question for future research.

The formalism of our theory of continuous spectrum expansions depends on the introduction of a locally convex space $W$ with certain properties, such that $H$ takes $W$ continuously into itself. It is needed in order to have a core where all operations make sense, and from which estimates may be extended by the closed graph theorem. It also allows us to say what an eigenfunction is; it is just an element of $W^{\prime}$ such that $H^{\prime} F=\lambda F$. This, together with regularity theorems for the domain of $\mathrm{H}$, if $\mathrm{H}$ is a differential operator, is what turns an abstractly defined eigenfunction into a concrete object such as a smooth function. For example, if $W=C_{0}^{\infty}(\Omega)$, and $\Omega$ is an open subset of a $\mathrm{C}^{\infty}$ manifold, and $\mathrm{H}$ is generated by a hypoelliptic differential expression, then $W^{\prime}$ is the space of distributions, so that if $F \in W^{\prime}$ and $H^{\prime} F=\lambda F$, then $F$ is a $C^{\infty}$ function. If the operator $\mathrm{H}$ is, for example, associated with a Dirichlet problem, smootheness of $\mathrm{F}$ is needed to show that $F$ vanishes on the boundary of $\Omega$. It should be noted that the smoothness of $F$ does not follow only from the fact that $F \in W^{\prime}$, which is implied by virtually any theory, but from this fact together with the fact that $\mathrm{H}^{\prime} \mathrm{F}=\lambda \mathrm{F}$. Of course, there are many approaches to these expansions which imply, for example for Schrödinger operators, that the generalized eigenfunctions satisfy the differential equation in a distributional sense and hence classically, but these assertions are shown as consequences of specific properties of the examples being studied. Since such assertions are necessary for applications, we build them into the theory, producing a more powerful structure.

Motivated by the above discussion, in examples studied in this paper we often take $\mathrm{W}$ to be $C_{0}^{\infty}(\Omega)$; this causes us to assume smoothness of the coefficients when $H$ is a differential operator.

However, other choices of $\mathrm{W}$ would perhaps allow more general coefficients. This is another subject for further work.

The author has been fortunate enough to have many discussions of this theory with many different mathematicians over a period of some years. He would like especially to thank Christer Bennewitz, Rainer Hempel, and Don Hinton, although discussions with a number of others have also been very helpful. He would also like to thank W. D. Evans, University College, Cardiff, and the British SERC for support during the author's very pleasant four-month stay in Cardiff in the spring of 1987, when this paper was begun, and Peter Hislop and the University of Kentucky for their hospitality in the Spring semester of 1991, when the research for the paper was finished.

1. BASIC FORMALISM AND $\mathrm{L}_{2}$ ESTIMATES

In this section we develop the basic formalism and eigenvector estimates for our theory. We shall need to introduce some basic spaces $W$ and $W^{\prime}$. W is contained in the domain of the self-adjoint operator $H$, and $W^{\prime}$ is its dual space under a certain topology. One may think of $W$ as like $C_{0}^{\infty}\left(\mathbb{R}^{n}\right)$ and $W^{\prime}$ as the space of distributions on $\mathbb{R}^{n}$; also one may sometimes wish to think of $\mathrm{W}$ as the rapidly decreasing functions and $\mathrm{W}^{\prime}$ as the tempered distributions. In order to handle the case where $\mathrm{W}=\mathrm{C}_{0}^{\infty}\left(\mathbb{R}^{\mathrm{n}}\right)$, we need to assume that $\mathrm{W}$ is an inductive limit of Frechet spaces, rather than a Frechet space itself, since $C_{0}^{\infty}\left(\mathbb{R}^{\mathrm{n}}\right)$ under the usual topology is not metrisable. (See Proposition 5, p. 125, Robertson and Robertson [7].) The purpose of these topological vector spaces is to get a precise definition of what the eigenfunctions are: they are just elements $F$ of $W^{\prime}$ such that $H^{\prime} F=\lambda F$ for some $\lambda$, where $H^{\prime}$ is the transpose of $H$. The structure of $W$ is needed for 
an application of the closed graph theorem to obtain a priori estimates from assertions about the domain of $\mathrm{H}$.

Notation 1.1. Let $\mathfrak{h}$ be a Hilbert space, and let $H$ be a self-adjoint operator with domain a dense subspace of $\mathfrak{h}$ and range contained in $\mathfrak{h}$. If $e \in \mathfrak{h}$, let $S_{e}$ denote the closed linear span of $\{\mathrm{P}(\Delta) \mathrm{e} \mid \Delta$ is a Borel subset of $\mathbb{R}\}$, where for any Borel set $\Delta, \mathrm{P}(\Delta)$ is the spectral projection associated with $\Delta$ by the spectral theorem. Let $\sigma_{\mathrm{e}}(\Delta)=[\mathrm{P}(\Delta) \mathrm{e}, \mathrm{e}]$, where $[$,$] denotes the inner$ product of $\mathfrak{h}$. (In this paper, $\mathfrak{h}$ will always be $\mathrm{L}_{2}(\mathrm{X}, \rho)$, where $\rho$ is a positive measure on $\mathrm{X}$ ). Note that $\sigma_{\mathrm{e}}$ is a positive Borel measure on $\mathbb{R}$, such that $\sigma_{\mathrm{e}}(\mathbb{R})=\|\mathrm{e}\|^{2}$. Note also that the restriction of $H$ to $S_{e}$ is a self-adjoint operator which is unitarily equivalent to the operator in $L_{2}\left(\sigma_{e}\right)$ which maps $f(\lambda)$ to $\lambda f(\lambda)$.

Assumption 1.2. Throughout the paper, we shall assume the following hypotheses:

i) $H$ is a self-adjoint operator with domain a dense subset of $\mathfrak{h}$ and range contained in $\mathfrak{h}=\mathrm{L}_{2}(\mathrm{X}, \rho)$, where $\mathrm{X}$ is a locally compact Hausdorff space, and $\rho$ is a positive regular Borel measure on $\mathrm{X}$ such that the measure of every compact set is finite;

ii) $W$ is the inductive limit of a sequence $\left\{V_{n}\right\}$ of separable Frechet spaces such that for each $n, V_{n}$ is algebraically and topologically contained in or equal to $V_{n+1}$; (hence $\mathrm{W}$ is complete, by Prop. 3, p.128, [5]); (note that a subbase for the topology of $\mathrm{W}$ is the set of all absolutely convex subsets $U$ of $W$ such that $U \cap V_{n}$ is open in $V_{n}$ for every $n$; recall that $W$ is metrisable if and only if for some $M, V_{n}=V_{M}$ for $n \geq M$, by Prop. 5, p. 129, [7]);

iii) $\mathbf{W}^{\prime}$ is the dual of $\mathbf{W}$, and $\mathbf{W}^{\prime}$ is given the topology $\sigma\left(\mathbf{W}^{\prime}, \mathbf{W}\right)$ of pointwise convergence on $W$; (recall that a neighborhood subbase about 0 for this topology is the set of neighborhoods $\mathrm{U}(\mathrm{x}, \epsilon)=\{\mathrm{F}:|\mathrm{F}(\mathrm{x})|<\epsilon\})$;

iv) $\mathrm{W}$ C domain $\mathrm{H}$ and $\mathrm{H}$ is a continuous linear transformation from $\mathrm{W}$ into $\mathrm{W}$;

v) $W$ is contained and dense in $\mathrm{L}_{1}(\mathrm{X}, \rho) \cap \mathrm{L}_{2}(\mathrm{X}, \rho)$ and the identity mapping from $\mathrm{W}$ into $\mathrm{L}_{1}(\mathrm{X}, \rho)$ and $\mathrm{L}_{2}(\mathrm{X}, \rho)$ is continuous;

vi) for any open set $\Gamma$ in $X$, if $C_{c}(X)$ denotes the continuous complex-valued functions of compact support in $X$, and if $\phi$ is any element in $C_{C}(X)$ which is supported in $\Gamma$, there is a sequence $\left\{\phi_{n}\right\}$ of elements of $W$, each supported in $\Gamma$, such that $\phi_{n}$ converges in $L_{2}(X, \rho)$ to $\phi$.

Remark: We shall sometimes assume the following estimate; we shall explicitly state this assumption each time.

Estimate 1.3. (an a priori estimate) There exists a 1-1 continuous linear transformation B from $W$ onto $W$ and a positive function $f \in L_{2}(X, \rho)$, such that multiplication by $f$ maps $W$ continuously into $\mathrm{W}$ and such that the linear transformation $\mathrm{B}^{\prime}$ has the property that there exists a positive integer $N$ such that $\left\{B^{\prime} \phi\right\} / f \in L_{\infty}(X, \rho)$ for all $\phi$ in the domain of $H^{N}$. (Note that by $v$ ) of Assumption 1.2, $\mathrm{L}_{2}(\mathrm{X}, \rho)$ is naturally embedded in $\left.\mathrm{W}^{\prime}\right)$.

Remark: We now make the initial definition of the eigenfunctions, as linear functionals on a dense subspace of $\mathrm{W}$ over the rationals. The work consists of showing that they belong to a natural space. Note that $\mathrm{W}$ has a countable dense set by hypothesis ii) above. Let $\mathrm{S}^{\prime}$ be a countable dense subspace of $\mathrm{W}$ over the rationals. 
Notation: Let $\mathrm{e} \in \mathrm{h}$. Let $\phi_{\mathrm{e}}$ be the unitary mapping from $\mathrm{S}_{\mathrm{e}}$ onto $\mathrm{L}_{2}\left(\sigma_{\mathrm{e}}\right)$, such that $\phi_{\mathrm{e}}(\mathrm{e})=1$, and such that $\phi_{\mathrm{e}}(\mathrm{H} \phi)(\lambda)=\lambda \phi_{\mathrm{e}}(\phi)(\lambda)$, for all $\phi$ in $\mathrm{D}(\mathrm{H}) \cap \mathrm{S}_{\mathrm{e}}$, and such that $\phi_{\mathrm{e}}(\mathrm{P}(\Delta) \phi)=\chi(\Delta) \phi_{\mathrm{e}}(\phi)$, where $\chi(\Delta)$ denotes the characteristic function of the Borel set $\Delta$. Let $\mathrm{U}_{\mathrm{e}}=\phi_{\mathrm{e}} \circ \mathrm{P}\left(\mathrm{S}_{\mathrm{e}}\right)$, where $\mathrm{P}\left(\mathrm{S}_{\mathrm{e}}\right)$ denotes the orthogonal projection onto $\mathrm{S}_{\mathrm{e}}$. Note that the existence and uniqueness of $\phi_{e}$ follows from the spectral theorem. Let $S=S^{\prime}+H S^{\prime}$. For each $\phi_{i} \in S$, select an everywhere defined representative of $U_{e}\left(B \phi_{i}\right)$. Denote the above representative by $g_{i}$.

Definition 1.4. For each $\lambda \in \mathbb{R}$, define $\hat{\mathrm{Z}}_{\lambda, \mathrm{e}}$ on $\mathrm{S}$ by $\hat{\mathrm{Z}}_{\lambda, \mathrm{e}}\left(\phi_{\mathrm{i}}\right)=\mathrm{g}_{\mathrm{i}}(\lambda)$, so that if $\mathrm{U}_{\mathrm{e}}$ is the mapping discussed above which arises from the spectral theorem, then $\hat{Z}_{\lambda, e}\left(\phi_{i}\right)=U_{e}\left(B \phi_{i}\right)(\lambda)$ for almost every $\lambda$ with respect to $\sigma_{e}$. Note that, if $\Delta_{0}$ is the complement of the set of $\lambda$ such that $\hat{\mathrm{Z}}_{\lambda, \mathrm{e}}$ is a linear functional on $\mathrm{S}$ over the rationals, then $\sigma_{\mathrm{e}}\left(\lambda_{0}\right)=0$.

Lemma 1.5. If there exists a positive constant $\mathrm{M}$ such that $\left\|\left(\mathrm{B}^{\prime} \theta\right) / \mathrm{f}\right\|_{\infty} \leq \mathrm{M}\left(\|\theta\|_{2}^{2}+\left\|\mathrm{H}^{\mathrm{N}} \theta\right\|_{2}^{2}\right)^{1 / 2}$ for all $\theta$ in the domain of $\mathrm{H}^{\mathrm{N}}$, where $\mathrm{f}$ is as in Estimate 1.3, then for almost every $\mathbf{x}$ with respect to $\rho$ the following is true: for any orthogonal set $\left\{\mathrm{e}_{\mathrm{i}}\right\}$ in the domain of $\mathrm{H}^{\mathrm{N}}$, such that $\mathrm{He}_{\mathrm{i}}$ is also an orthogonal set, and such that $\left\|\mathrm{e}_{\mathrm{i}}\right\|^{2}+\left\|\mathrm{H}^{\mathrm{N}} \mathrm{e}_{\mathrm{i}}\right\|^{2}=1$ for all $\mathrm{i}$, then $\left(\Sigma_{\mathrm{i}=1}^{\infty}\left|\mathrm{B}^{\prime} \mathrm{e}_{\mathrm{i}}\right|^{2}(\mathrm{x})\right)^{1 / 2} \leq \mathrm{Mf}(\mathrm{x})$.

Proof: It can be proved by a technique like that of Weidmann [9], p. 140, that if $\mathrm{T}$ is a bounded operator from a separable Hilbert space $h$ into $L_{\infty}(X, \rho)$, and $\|T\|$ is the associated operator norm, then for almost every $\mathbf{x}$ with respect to $\rho$, it is true that for any orthonormal set $\left\{\mathrm{e}_{\mathrm{i}}\right\}$ in $\mathrm{h},\left(\Sigma_{\mathrm{i}}\left|\mathrm{Te}_{\mathrm{i}}(\mathrm{x})\right|^{2}\right)^{1 / 2} \leq\|\mathrm{T}\|$. The idea of the proof is to construct a mapping $\mathrm{Q}$ from $\mathrm{X}$ into $h$ such that $\operatorname{Tg}(\mathbf{x})=[Q(x), g]$. Then one can see from the hypothesis that for almost every $\mathbf{x}, Q(\mathbf{x})$ has norm less than or equal to $\|T\|$. Then for each fixed $x$, the Schwartz inequality gives the desired result. To make this proof rigorous demands careful attention to sets of measure 0 with respect to $\rho$. Now if we let $\mathrm{Tg}$ be $\left(\mathrm{B}^{\prime} \mathrm{g}\right) / \mathrm{f}$, and $\mathrm{h}$ be the domain of $\mathrm{H}^{\mathrm{N}}$ with norm $\|g\|_{h}^{2}=\|g\|_{2}^{2}+\left\|\mathrm{H}^{\mathrm{N}} \mathrm{g}\right\|_{2}^{2}$, the result follows.

Another way to construct the mapping $Q$ which does not pay such careful attention to sets of measure 0 is due to $\mathrm{C}$. Bennewitz and the author:

Note that by the Gelfand representation theorem for commutative Banach algebras $\mathrm{L}_{\omega}(\mathrm{X}, \rho)$ is isomorphic and isometric as a Banach algebra to the algebra $\mathrm{C}(\mathrm{Y})$, where $\mathrm{Y}$ is the maximal ideal space of the Banach algebra $\mathrm{L}_{\infty}(\mathrm{X}, \rho)$, a compact Hausdorff space. Let $\mathrm{E}$ be the isometry from $\mathrm{L}_{\infty}(\mathrm{X}, \rho)$ onto $\mathrm{C}(\mathrm{Y})$. Define $\hat{\mathrm{T}}$ to be the operator ET. Then at every $\mathrm{y} \in \mathrm{Y}$, define $\hat{\mathrm{Q}}_{\mathrm{y}}(\mathrm{g})$ to be $\operatorname{ETg}(\mathrm{y})$. It is clear from the hypotheses that for all $y \in Y$, the linear functional $\hat{Q}_{y}$ is in the dual space of $h$, and has norm less than or equal to $M=\|T\|$. Thus $\hat{Q}_{y}(g)=[g, \alpha]$ for some $\alpha \in \mathrm{h}$, with $\|\alpha\| \leq \mathrm{M}$. It follows that for each orthonormal set $\left\{\mathrm{e}_{\mathrm{i}}\right\}$ of elements of $\mathrm{h}$, and for each finite $\mathrm{N},\left(\Sigma_{\mathrm{i}=1}^{\mathrm{N}}\left|\mathrm{ETe}_{\mathrm{i}}(\mathrm{y})\right|^{2}\right)^{1 / 2} \in \mathrm{C}(\mathrm{Y})$ as a function of $\mathrm{y}$, with supremum norm less than or equal to $M$. Hence for each $N,\left(\Sigma_{i=1}^{N}\left|T_{i}(x)\right|^{2}\right)^{1 / 2} \in L_{\infty}(X, \rho)$ as a function of $x$ with $L_{\infty}$ norm less than or equal to $\mathrm{M}$, since the map $\mathrm{E}$ is an isometric isomorphism and takes absolute values to 
absolute values. It follows immediately that for almost every $\mathbf{x}$ in $\mathrm{X}$ with respect to $\rho$, $\left(\Sigma_{\mathrm{i}=1}^{\infty}\left|\mathrm{Te}_{\mathrm{i}}(\mathrm{x})\right|^{2}\right)^{1 / 2} \leq \mathrm{M}$, as we desired to show.

Lemma 1.6. Let $\mathrm{n}$ be a positive integer. Assume the hypotheses of Estimate 1.9. Then the following hold:

i) $\mathrm{B}$ is a homeomorphism from $\mathrm{W}$ onto $\mathrm{W}$, and $\mathrm{B}$ ' is a homeomorphism from $\mathrm{W}$ ' onto $\mathrm{W}$ '.

ii) There exists a constant $\mathrm{K}$ such that for any $\phi \in \operatorname{domain} \mathrm{H}^{\mathrm{N}}$,

$$
\left\|\left(\mathrm{B}^{\prime} \phi\right) / \mathrm{f}\right\|_{\infty} \leq \mathrm{K}\left(\|\phi\|_{2}+\left\|\mathrm{H}^{\mathrm{N}} \phi\right\|_{2}\right) \text {. }
$$

iii) Let $\{\xi(\mathrm{i})\}_{\mathrm{i}=1}^{\mathbf{k}}$ be any pairwise disjoint collection of Borel subsets of $\mathbb{R}$; let $\mathrm{K}$ be as in ii) above. Suppose that $\mathrm{e}$ is in the domain of $\mathrm{H}^{\mathrm{N}}$, and that $\left\{\theta_{\mathrm{i}}\right\}$ is any collection of elements of $W$ such that $\left\|\theta_{\mathrm{i}}\right\|_{2} \leq 1$. Then

$$
\Sigma_{\mathrm{i}=1}^{\mathrm{k}} \int_{\xi(\mathrm{i})}\left|\mathrm{U}_{\mathrm{e}} \mathrm{B} \theta_{\mathrm{i}}\right| \mathrm{d} \sigma_{\mathrm{e}}(\lambda) \leq \sqrt{2} \mathrm{~K}\|\mathrm{~N}(\Gamma) \mathrm{f}\|_{2}\|\mathrm{e}\|_{\mathrm{h}^{\prime}}
$$

where $\|\mathrm{e}\|_{\mathrm{h}}^{2}=\|\mathrm{e}\|_{2}^{2}+\left\|\mathrm{H}^{\mathrm{N}} \mathrm{e}\right\|_{2}^{2}, \Gamma$ is the union of the supports of the functions $\theta_{\mathrm{i}}$, and $\mathrm{N}(\Gamma)$ is the characteristic function of $\Gamma$.

Proof: To prove the first conclusion, we note that the mapping $B$ is a 1-1 mapping from $\mathrm{W}$ onto itself. This implies that $\mathrm{B}$ is a homeomorphism, by a result of Dieudonne and Schwartz (see the discussion on page 124 of [7]). However, it is now easy to prove this result from later work on webs; for completeness we give the proof. It is clear that the graph of $\mathrm{B}^{-1}$ is closed. By Theorem 2, page 158, [7], we see that if $\mathrm{E}$ is a Frechet space, and $\mathrm{F}$ is a separated convex space with a completing web, then mappings from $\mathbf{E}$ onto $\mathrm{F}$ with (sequentially) closed graph are continuous. It is clear from the definition of a compatible web that a Frechet space or the strict inductive limit of Frechet spaces has a compatible web, which is completing by Lemma 1, p. 156, [7]. Hence, $\mathrm{W}$ has a completing web. To show the continuity of $\mathrm{B}^{-1}$, we need only show that the restriction of $B^{-1}$ to each space $V_{n}$ is continuous, since for an open set $U, B^{-1}(U)$ is the union of its intersection with each $V_{n}$, and since a set is open in $W$ if and only if its intersection with each $V_{n}$ is open. But each $V_{n}$ is a Frechet space, and it is obvious from the continuity of $B$ that the graph of the restriction of $B^{-1}$ to each $V_{n}$ is sequentially closed. Therefore $B^{-1}$ is continuous, as we desired to show. That $\mathrm{B}^{\prime}$ is a homeomorphism is immediate.

The second conclusion is a consequence of the closed graph theorem, because the mapping $\mathrm{T}$ taking the domain of $\mathrm{H}^{\mathrm{N}}$, with graph norm, into $\mathrm{L}_{\infty}(\mathrm{X}, \rho)$ has closed graph, where $\mathrm{T} \phi=\left\{\mathrm{B}^{\prime} \phi\right\} / \mathrm{f}$. To see this, suppose that $\phi_{\mathrm{n}}$ is a Cauchy sequence of elements of the domain of $\mathrm{H}^{\mathrm{N}}$, in graph norm, and that $\mathrm{T} \phi_{\mathrm{n}}$ is Cauchy in $\mathrm{L}_{\infty}(\mathrm{X}, \rho)$. Then $\phi_{\mathrm{n}}$ converges in $\mathrm{L}_{2}(\mathrm{X}, \rho)$ to an element $\phi$ of the domain of $\mathrm{H}^{\mathrm{N}}$. We must show that $\mathrm{T} \phi_{\mathrm{n}}$ converges to $\mathrm{T} \phi$. Since $\phi_{\mathrm{n}}$ converges to $\phi$ in $\mathrm{L}_{2}(\mathrm{X}, \rho)$, then $\phi_{\mathrm{n}}$ converges to $\phi$ in $\mathrm{W}^{\prime}$, where we have embedded $\mathrm{L}_{2}(\mathrm{X}, \rho)$ into $\mathrm{W}^{\prime}$ using $\left.\mathrm{v}\right)$ of Assumption 1.2 by mapping $g$ to the linear functional $F_{g}$ such that $F_{g}(\phi)=[\phi, g]$. Since $B^{\prime}$ is continuous from $W^{\prime}$ into $W^{\prime}$, then $B^{\prime} \phi_{n}$ converges in $W^{\prime}$ to $B^{\prime} \phi$. But by hypothesis, $\left(B^{\prime} \phi_{n}\right) / f$ is Cauchy in $\mathrm{L}_{\infty}(\mathrm{X}, \rho)$ and therefore converges to an element $\theta$ of $\mathrm{L}_{\infty}(\mathrm{X}, \rho)$. The preceding argument, using $v$ ) of Assumption 1.2 for $\mathrm{L}_{1}(\mathrm{X}, \rho)$, shows that convergence in $\mathrm{L}_{\infty}(\mathrm{X}, \rho)$ implies convergence in $\mathrm{W}^{\prime}$. However, multiplication by $\mathrm{f}$ takes $\mathrm{W}^{\prime}$ continuously into itself, where by definition 
$\mathrm{fF}(\phi)=\mathrm{F}(\mathrm{f} \phi)$, because the transpose of a continuous map from $\mathrm{W}$ into $\mathrm{W}$ is a continuous map from $W^{\prime}$ into $W^{\prime}$. But we have embedded $L_{2}(X, \rho)$ into $W^{\prime}$ using an embedding map $E$ such that $\mathrm{E}(\mathrm{f} \phi)=\mathrm{fE}(\phi)$. Thus $\mathrm{B}^{\prime} \phi_{\mathrm{n}}$ converges to $\mathrm{f} \theta$ in $\mathrm{W}^{\prime}$. Thus $\mathrm{B}^{\prime} \phi=\mathrm{f} \theta$ in $\mathrm{W}^{\prime}$. Hence $\mathrm{B}^{\prime} \phi(\mathrm{x})=\mathrm{f} \theta(\mathrm{x})$ for almost every $\mathrm{x}$ in $\mathrm{X}$ with respect to $\rho$. Therefore $\left(\mathrm{B}^{\prime} \phi\right) / \mathrm{f}=\theta$, as we desired to show. The second assertion is proved.

We prove the third assertion. Note that the second conclusion of the lemma guarantees that for any $\phi$ in the domain of $\mathrm{H}^{\mathrm{N}},\left\|\left(\mathrm{B}^{\prime} \phi\right) / \mathrm{f}\right\|_{\infty} \leq \sqrt{2} \mathrm{~K}\left(\|\phi\|_{2}^{2}+\left\|\mathrm{H}^{\mathrm{N}} \phi\right\|_{2}^{2}\right)^{1 / 2}$. We may therefore use $\sqrt{2} \mathrm{~K}$ as the constant $\mathrm{M}$ in Lemma 1.5. Define $b_{\mathrm{i}}$ by $\mathrm{b}_{\mathrm{i}}(\lambda)=\left|\mathrm{U}_{\lambda, \mathrm{e}}\left(\mathrm{B} \theta_{\mathrm{i}}\right)\right| / \mathrm{U}_{\mathrm{e}}\left(\mathrm{B} \theta_{\mathrm{i}}\right)$ if $\mathrm{U}_{\mathrm{e}}\left(\mathrm{B} \theta_{\mathrm{i}}\right) \neq 0$; define $\mathrm{b}_{\mathrm{i}}(\lambda)$ to be 0 otherwise. Note that each $\mathrm{b}_{\mathrm{i}}$ is measurable with respect to $\sigma_{\mathrm{e}}$. Use $\mathrm{r}^{*}$ to denote the complex conjugate of the function r. Let $\aleph(\xi(\mathrm{i}))$ denote the characteristic function of $\xi(\mathrm{i})$, and define $g \in \mathrm{S}_{e}$ by $\mathrm{U}_{\mathrm{e}}(\mathrm{g})=\Sigma_{\mathrm{i}=1}^{\mathrm{k}} \overline{\mathrm{b}}_{\mathrm{i}} \aleph(\xi(\mathrm{i}))$. By Lemma 1.5, we see that if $\mathrm{e}_{\mathrm{i}}=(\mathrm{P}(\xi(\mathrm{i})) \mathrm{g}) /\|\mathrm{P}(\xi(\mathrm{i})) \mathrm{g}\|_{\mathrm{h}}$, then $\Sigma_{\mathrm{i} 1}^{\mathrm{k}}\left|\mathrm{B}^{\prime} \mathrm{e}_{\mathrm{i}}(\mathrm{x})\right|^{2} \leq 2 \mathrm{~K}^{2} \mathrm{f}^{2}$ for almost every $\mathbf{x}$. Thus

$$
\begin{aligned}
& \Sigma_{\mathrm{i}=1}^{\mathrm{k}} \int_{\xi(\mathrm{i})}\left|\mathrm{U}_{\mathrm{e}}\left(\mathrm{B} \theta_{\mathrm{i}}\right)\right| \mathrm{d} \sigma_{\mathrm{e}}(\lambda)=\Sigma_{\mathrm{i}=1}^{\mathrm{k}} \int_{\xi(\mathrm{i})} \mathrm{b}_{\mathrm{i}}(\lambda) \mathrm{U}_{\mathrm{e}}\left(\mathrm{B} \theta_{\mathrm{i}}\right) \mathrm{d} \sigma_{\mathrm{e}}(\lambda)= \\
& \Sigma_{\mathrm{i}=1}^{\mathbf{k}} \int\left\{\mathrm{U}_{\mathrm{e}}\left(\mathrm{B} \theta_{\mathrm{i}}\right)\right\} \mathrm{U}_{\mathrm{e}}(\mathrm{P}(\xi(\mathrm{i})) \mathrm{g}\}^{*}(\lambda) \mathrm{d} \sigma_{\mathrm{e}}(\lambda)=\Sigma_{\mathrm{i}=1}^{\mathbf{k}}\left[\mathrm{B} \theta_{\mathrm{i}}, \mathrm{P}(\xi(\mathrm{i})) \mathrm{g}\right]= \\
& \Sigma_{\mathrm{i}=1}^{\mathrm{k}} \int_{\Gamma} \theta_{\mathrm{i}}(\mathrm{x})\left\{\mathrm{B}^{\prime} \mathrm{P}(\xi(\mathrm{i})) \mathrm{g}\right\}^{*}(\mathrm{x}) \mathrm{d} \rho(\mathrm{x}) \leq \Sigma_{\mathrm{i}=1}^{\mathrm{k}}\left\|\aleph(\Gamma) \mathrm{B}^{\prime} \mathrm{P}(\xi(\mathrm{i})) \mathrm{g}\right\|_{2}= \\
& \Sigma_{\mathrm{i}=1}^{\mathrm{k}}\|\mathrm{P}(\xi(\mathrm{i})) g\|_{\mathrm{h}}\left\|\aleph(\Gamma) \mathrm{B}^{\prime} \mathrm{e}_{\mathrm{i}}\right\|_{2} \leq\left(\Sigma_{\mathrm{i}=1}^{\mathrm{k}}\|\mathrm{P}(\xi(\mathrm{i})) g\|_{\mathrm{h}}^{2}\right)^{1 / 2}\left(\Sigma_{\mathrm{i}=1}^{\mathrm{k}}\left\|\aleph(\Gamma) \mathrm{B}^{\prime} \mathrm{e}_{\mathrm{i}}\right\|_{2}^{2}\right)^{1 / 2} .
\end{aligned}
$$

But $\Sigma_{\mathrm{i}=1}^{\mathrm{k}}\left\|\aleph(\Gamma) \mathrm{B}^{\prime} \mathrm{e}_{\mathrm{i}}\right\|_{2}^{2}=\Sigma_{\mathrm{i}=1}^{\mathrm{k}} \int_{\Gamma}\left|\mathrm{B}^{\prime} \mathrm{e}_{\mathrm{i}}\right|^{2} \mathrm{~d} \rho \leq 2 \mathrm{~K}^{2} \int_{\Gamma} \mathrm{f}^{2} \mathrm{~d} \rho$. Hence

$$
\Sigma_{\mathrm{i}=1}^{\mathrm{k}} \int_{\xi(\mathrm{i})}\left|\mathrm{U}_{\mathrm{e}}\left(\mathrm{B} \theta_{\mathrm{i}}\right)\right| \mathrm{d} \sigma_{\mathrm{e}}(\lambda) \leq \sqrt{2} \mathrm{~K}\left(\Sigma_{\mathrm{i}=1}^{\mathrm{k}}\|\mathrm{P}(\xi(\mathrm{i})) g\|_{\mathrm{h}}^{2}\right)^{1 / 2}\|\aleph(\Gamma) \mathrm{f}\|_{2} \leq \sqrt{2} \mathrm{~K}\|\mathrm{e}\|_{\mathrm{h}}\|\aleph(\Gamma) \mathrm{f}\|_{2}
$$

The third assertion follows immediately; the lemma is proved.

Corollary 1.7. Assume the hypotheses of Estimate 1.3. Use the notation of Definition 1.4. Suppose that $\mathrm{e}$ is in the domain of $\mathrm{H}^{\mathrm{N}}$. Then for every $\epsilon>0$ there exists an $\mathrm{M}>0$ such that $\sigma_{\mathrm{e}}\left(\Delta_{\mathrm{M}}\right)<\epsilon$, where $\Delta_{\mathrm{M}}=\left\{\lambda \in \mathbb{R} \backslash \Delta_{0} \mid \exists \phi \in \mathrm{S}\right.$ with $\left.\left|\hat{\mathrm{Z}}_{\lambda, \mathrm{e}}(\mathrm{B} \phi)\right| \geq \mathrm{M}\|\phi\|_{2}\right\}$.

Proof: Let us write the countable set $\mathrm{S}$ as $\left\{\phi_{\mathrm{i}}\right\}$. Let $\xi_{\mathrm{iM}}=\left\{\lambda|| \hat{\mathrm{Z}}_{\lambda, \mathrm{e}}\left(\mathrm{B} \phi_{\mathrm{i}}\right) \mid \geq \mathrm{M}\left\|\phi_{\mathrm{i}}\right\|_{2}\right.$. Let $\theta_{\mathrm{i}}=\phi_{\mathrm{i}} / \mathrm{r}_{\mathrm{i}}$, where $\mathrm{r}_{\mathrm{i}}$ is a rational number such that $\left\|\phi_{\mathrm{i}}\right\|_{2} \leq \mathrm{r}_{\mathrm{i}} \leq 2\left\|\dot{\phi}_{\mathrm{i}}\right\|_{2}$. For any fixed $\mathrm{M}$, let $\eta_{1}=\xi_{1 \mathrm{M}}$ and $\eta_{\mathrm{j}}=\xi_{\mathrm{jM}} \backslash U_{\mathrm{r}=1}^{\mathrm{j}-1} \xi_{\mathrm{rM}}$. Then for any $\mathbf{k}$,

$$
\Sigma_{\mathrm{i}=1}^{\mathrm{k}} \int_{\eta_{\mathrm{i}}}\left|\mathrm{U}_{\mathrm{e}}\left(\mathrm{B} \theta_{\mathrm{i}}\right)\right| \mathrm{d} \sigma_{\mathrm{e}}(\lambda) \geq(\mathrm{M} / 2) \sigma_{\mathrm{e}}\left(\mathrm{U}_{\mathrm{i}=1}^{\mathrm{k}} \eta_{\mathrm{i}}\right)
$$

By Lemma 1.6, we see that

$$
\sigma_{\mathrm{e}}\left(\mathrm{U}_{\mathrm{i}=1}^{\mathrm{k}} \eta_{\mathrm{i}}\right) \leq(2 / \mathrm{M}) \sqrt{2} \mathrm{~K}\|\mathrm{f}\|_{2}\left(\left\|\mathrm{P}\left(\mathrm{u}_{\mathrm{i}=1}^{\mathrm{k}} \eta_{\mathrm{i}}\right) \mathrm{e}\right\|_{2}^{2}+\left\|\mathrm{H}^{\mathrm{N}} \mathrm{P}\left(\mathrm{u}_{\mathrm{i}=1}^{\mathrm{k}} \eta_{\mathrm{i}}\right) \mathrm{e}\right\|_{2}^{2}\right)^{1 / 2}
$$

The corollary is proved.

Theorem 1.8. Assume the hypotheses of Estimate 1.9. Suppose that $\mathrm{e}$ is in the domain of $\mathrm{H}^{\mathrm{N}}$, and that \|\|$_{\mathrm{h}}$ is as in Assertion iii) of Lemma 1.6. Then there exists a subset $\zeta$ of $\mathbb{R}$ such that $\sigma_{\mathrm{e}}(\mathbb{R} \backslash \zeta)=0$ and such that for every $\lambda \in \zeta$, there exists a unique element $\mathrm{Z}_{\lambda, \mathrm{e}}$ in $\mathrm{W}^{\prime}$ such that $\mathrm{Z}_{\lambda, \mathrm{e}}$ is not the zero functional and $\mathrm{Z}_{\lambda, \mathrm{e}}$ agrees with $\hat{\mathrm{Z}}_{\lambda, \mathrm{e}}$ on $\mathrm{S}$. Furthermore, if $\mathrm{F}_{\lambda, \mathrm{e}}$ is defined on $\zeta$ by the relation $\mathrm{F}_{\lambda, \mathrm{e}}=\left(\mathrm{B}^{\prime}\right)^{-1} \mathrm{Z}_{\lambda, \mathrm{e}}$, then: 
i) $\mathrm{H}^{\prime} \mathrm{F}_{\lambda, \mathrm{e}}=\lambda \mathrm{F}_{\lambda, \mathrm{e}}$, where $\mathrm{H}^{\prime}$ is the conjugate of the restriction of $\mathrm{H}$ to $\mathrm{W}$; furthermore, for any $\theta \in \mathrm{W}, \mathrm{F}_{\beta, \mathrm{e}^{(\theta)}}=\mathrm{U}_{\mathrm{e}}(\theta)(\beta)$ for almost every $\beta$ with respect to $\sigma_{\mathrm{e}}$;

ii) $\mathrm{B}^{\prime} \mathrm{F}_{\lambda, \mathrm{e}} \in \mathrm{L}_{2}(\mathrm{X}, \rho)$;

iii) if $\alpha(\lambda)=\mathrm{B}^{\prime} \mathrm{F}_{\lambda, \mathrm{e}^{\prime}}$, then $\alpha$ is a measurable function with respect to $\sigma_{\mathrm{e}}$ from $\mathbb{R}$ into $\mathrm{L}_{2}(\mathrm{X}, \rho)$, in the sense that $\forall \epsilon>0$ there exists a compact set $\Gamma$ such that $\sigma_{\mathrm{e}}(\mathbb{R} \backslash \Gamma)<\epsilon$ and such that the restriction of $\alpha$ to $\Gamma$ is a continuous function from $\Gamma$ into $\mathrm{L}_{2}(\mathrm{X}, \rho)$;

iv) if $\beta$ is a Borel subset of $\mathrm{X}$ and $\Delta$ is a Borel subset of $\mathbb{R}$, then $\int_{\Delta}\|\chi(\beta) \alpha(\lambda)\|_{2} \mathrm{~d} \sigma_{\mathrm{e}} \leq \sqrt{2} \mathrm{~K}\|\mathrm{P}(\Delta) \mathrm{e}\|_{\mathrm{h}}\|\chi(\beta)\|_{2}$, where $\chi(\beta)$ is the characteristic function of $\beta$.

Proof: $\mathrm{B}^{\prime}$ is a homeomorphism from $\mathrm{W}^{\prime}$ onto $\mathrm{W}^{\prime}$. By the preceding lemma, for almost every $\lambda$ the functional $\hat{Z}_{\lambda, e}$ has a unique extension $Z_{\lambda, e}$ to $W$. There exists a unique element of $\mathrm{L}_{2}(\mathrm{X}, \rho)$ which agrees with $\mathrm{Z}_{\lambda, \mathrm{e}}$ on $\mathrm{W}$; denote this also by $\mathrm{Z}_{\lambda, \mathrm{e}}$. For any element $\phi$ of $\mathrm{S}^{\prime}$,

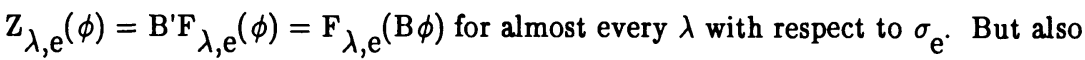
$\mathrm{Z}_{\lambda, \mathrm{e}}(\phi)=\mathrm{U}_{\mathrm{e}}(\mathrm{B} \phi)(\lambda)$ for almost every $\lambda$.

If $\delta$ is the set of $\lambda$ such that $\mathrm{Z}_{\lambda, \mathrm{e}}$ is the zero functional, then for every $\phi$ in $\mathrm{W}, \mathrm{U}_{\mathrm{e}}(\phi)$ vanishes on $\delta$. By continuity of $\mathrm{U}_{\mathrm{e}}$ from $\mathrm{L}_{2}(\mathrm{X}, \rho)$ into $\mathrm{L}_{2}\left(\sigma_{\mathrm{e}}\right)$, and since $\mathrm{W}$ is dense in $\mathrm{L}_{2}$, it follows that $\mathrm{U}_{\mathrm{e}}(\mathrm{e})$ vanishes on $\delta$. But this function is identically equal to 1 almost everywhere with respect to $\sigma_{\mathrm{e}}$. Hence $\sigma_{\mathrm{e}}(\delta)=0$. Thus $\mathrm{Z}_{\lambda, \mathrm{e}}$ is non-zero for almost every $\lambda$.

By the spectral theorem for self-adjoint operators in a Hilbert space, if $\mathrm{B} \phi=\theta$, we see

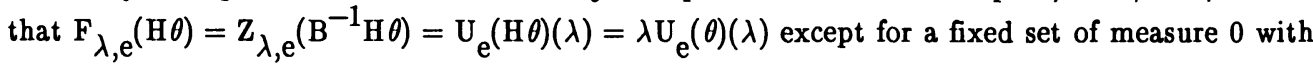
respect to $\sigma_{\mathrm{e}}$, which is the union of exceptional sets for each $\phi$ in the countable set $\mathrm{S}^{\prime}$. Since $F_{\lambda, e} \in W^{\prime}$, and since the range of the restriction of $B$ to $S^{\prime}$ is dense in the range of $B$ and therefore dense in $\mathrm{W}$, the first conclusion follows immediately. The second assertion was proved at the outset.

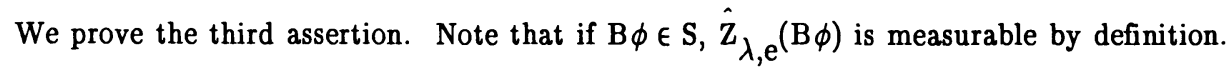
Extending by continuity, for almost every $\lambda$, to $L_{2}(X, \rho)$, we see that $\left[\phi, B^{\prime} F_{\lambda, e}\right]$ is a measurable function of $\lambda$ for every $\phi \in \mathrm{L}_{2}(\mathrm{X}, \rho)$. By proposition 8.15.2, p. 574, Edwards [3], the conclusion follows.

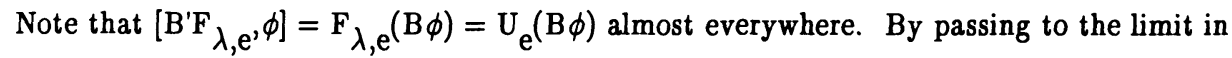
Lemma 1.6 we obtain that for any open set $\Gamma$ in $X$, and any partition $\{\xi(\mathrm{i})\}$ of $\Delta$, and any set of elements $\phi_{\mathrm{i}} \in \mathrm{L}_{2}(\mathrm{X}, \rho)$, each supported in $\Gamma$, such that $\left\|\phi_{\mathrm{i}}\right\|=1$,

$$
\Sigma_{\mathrm{i}=1}^{\mathbf{k}} \jmath_{\Delta}\left|\left[\mathrm{B}^{\prime} \mathrm{F}_{\lambda, \mathrm{e}^{,}} \phi_{\mathrm{i}}\right]\right| \mathrm{d} \sigma_{\mathrm{e}} \leq \sqrt{2 \mathrm{~K}}\|\chi(\Gamma) \mathrm{f}\|_{2}\left\|\mathrm{P}\left(\mathrm{U}_{\mathrm{i}} \xi(\mathrm{i})\right) \mathrm{e}\right\|_{\mathrm{h}} .
$$

Now use assertion iii) to select for every $\epsilon>0$ a compact set $\kappa_{\epsilon}$ of $\Delta$ such that $\sigma_{\mathrm{e}}\left(\Delta \mid \kappa_{\epsilon}\right)<\epsilon$ and such that the restriction of $\mathrm{B}^{\prime} \mathrm{F}_{\lambda, \mathrm{e}}$ to $\kappa_{\epsilon}$ is a continuous function from $\kappa_{\epsilon}$ into $\mathrm{L}_{2}(\mathrm{X}, \rho)$. It follows that, if \|\|$_{2, \Gamma}$ denotes the norm of $\mathrm{L}_{2}(\Gamma, \rho)$,

$$
\int_{\kappa_{\epsilon}}\left\|\mathrm{B}^{\prime} \mathrm{F}_{\lambda, \mathrm{e}}\right\|_{2, \Gamma} \mathrm{d} \sigma_{\mathrm{e}} \leq \sqrt{2} \mathrm{~K}\|\chi(\Gamma) \mathrm{f}\|_{2}\left\|\mathrm{P}\left(\kappa_{\epsilon}\right) \mathrm{e}\right\|_{\mathrm{h}} .
$$


From the monotone convergence theorem, it follows that

$$
\int_{\Delta}\left\|\mathrm{B}^{\prime} \mathrm{F}_{\lambda, \mathrm{e}}\right\|_{2, \Gamma} \mathrm{d} \sigma_{\mathrm{e}} \leq \sqrt{2} \mathrm{~K}\|\chi(\Gamma) \mathrm{f}\|_{2}\|\mathrm{P}(\Delta) \mathrm{e}\|_{\mathrm{h}} .
$$

Selecting a countable decreasing chain of open sets $\Gamma$ with intersection essentially equal to $\beta$, and using the monotone convergence theorem again, we easily complete the proof of iv), and hence the proof of Theorem 1.8 is completed.

\section{SOME REPRESENTATIVE EXAMPLES}

In this section, we take a look at some representative examples to motivate the theory.

Example 2.1. Let $\mathrm{X}=\mathbb{R}^{\mathrm{n}}$ and $\rho$ be Lebesgue measure. Let $\mathrm{W}$ denote $\mathrm{C}_{0}^{\infty}\left(\mathbb{R}^{\mathrm{n}}\right)$. It is well-known (see p. 75, [7]) that $W$ satisfies the hypotheses above. Suppose that $H$ is a self-adjoint operator in $\mathrm{L}_{2}\left(\mathbb{R}^{\mathrm{n}}\right)$ such that $\mathrm{H} \psi=\tau \psi$ for all $\psi$ in the domain of $\mathrm{H}$, where $\tau$ is a partial differential expression with $\mathrm{C}^{\boldsymbol{D}}$ coefficients. Suppose further that for some positive integer $N$, all elements of the domain of $H^{N}$ lie in $L_{\infty}\left(\mathbb{R}^{n}\right)$. Let $\omega$ be any bounded, $L_{2}$, positive element of $C^{\infty}\left(\mathbb{R}^{\mathrm{n}}\right)$. Let $\mathrm{B}$ be multiplication by $\omega$; then $\mathrm{B}^{\prime}$ is also multiplication by $\omega$. Let $\mathrm{f}$ in Estimate 1.3 be $\omega$. Theorem 1.8 then yields that, for any e in the domain of $H^{N}$, the eigenfunction $F_{\lambda, e}$ has the property that $\omega F_{\lambda, e}$ is an $L_{2}$ function, which has the properties of $B^{\prime} F_{\lambda, e}$ in this theorem. Here $\tau \mathrm{F}_{\lambda, \mathrm{e}}=\lambda \mathrm{F}_{\lambda, \mathrm{e}}$ in the sense of distributions.

Example 2.2. Suppose that $\mathrm{H}$ is a self-adjoint operator in $\mathrm{L}_{2}\left(\mathbb{R}^{\mathrm{n}}\right)$ such that $\mathrm{Hf}=\eta \mathrm{f}$ for all $\mathrm{f}$ in the domain of $\mathrm{H}$, where $\eta$ is a partial differential expression with $\mathrm{C}^{\infty}$ coefficients such that each derivative of any coefficient of $\eta$ has at most polynomial growth at infinity. Suppose that $\mathrm{H}$ has the additional property that for any positive integer $j$, there exists a positive integer $N(j)$ such that the domain of $\mathrm{H}^{\mathrm{N}(\mathrm{j})}$ is contained in the Sobolev space $\mathrm{H}^{\mathrm{j}}\left(\mathbb{R}^{\mathrm{n}}\right)$. Let $\tau$ denote the differential expression defined by $\tau=\Sigma_{\mathrm{i}=1}^{\mathrm{n}} \partial^{2} / \partial \mathrm{x}_{\mathrm{i}}^{2}-1$. Let $\mathrm{B}$ be the operator on $\mathrm{W}$ defined by $\mathrm{B} \phi=\left(|\mathbf{x}|^{2}+1\right)^{-(\mathrm{n}+\epsilon) / 4} \tau^{\mathrm{r}} \phi$, where $\mathrm{r}$ is large enough such that for any $\mathrm{g}$ such that $\mathrm{g} \in \mathrm{H}^{2 \mathrm{r}}\left(\mathbb{R}^{\mathrm{n}}\right)$, it follows that $g \in L_{\infty}\left(\mathbb{R}^{n}\right)$. Let $W$ be the space of rapidly decreasing functions on $\mathbb{R}^{n}$. By using the Fourier transform, we see that B satisfies the hypotheses of Estimate 1.3. W satisfies Assumption 1.2 since it is a Frechet space. Direct calculation shows that $\left.\mathrm{B}^{\prime}(\theta) /\left(|\mathrm{x}|^{2}+1\right)^{-(\mathrm{n}+\epsilon) / 4}\right) \in \mathrm{L}_{\infty}\left(\mathbb{R}^{\mathrm{n}}\right)$ for all $\theta$ in the domain of $\mathrm{H}^{\mathrm{N}(\mathrm{j})}$, where $\mathrm{j}$ is large enough that for $\theta$ in $\mathrm{H}^{\mathrm{j}}\left(\mathbb{R}^{\mathrm{n}}\right), \mathrm{D}^{\alpha} \theta \in \mathrm{L}_{\infty}\left(\mathbb{R}^{\mathrm{n}}\right)$ for $|\alpha| \leq 2$ r. By Theorem 1.8, we see that $\tau^{\mathrm{r}}\left\{\left(|x|^{2}+1\right)^{-(\mathrm{n}+\epsilon) / 4} \mathrm{~F}_{\lambda, \mathrm{e}}\right\} \in \mathrm{L}_{2}\left(\mathbb{R}^{\mathrm{n}}\right)$. Since $\mathrm{F}_{\lambda, \mathrm{e}}$ is in $W^{\prime}$, the space of tempered distributions, we see from the ordinary Fourier transform that $\left(|x|^{2}+1\right)^{-(n+\epsilon) / 4} F_{\lambda, e} \in L_{\infty}\left(\mathbb{R}^{n}\right)$.

Example 2.3. Let $\mathrm{H}$ be any self-adjoint operator in $\mathrm{L}_{2}\left(\mathbb{R}^{\mathrm{n}}\right)$ such that $\mathrm{H} \theta=\eta \theta$ for all $\theta$ in the domain of $\mathrm{H}$, where $\eta$ is a partial differential expression with $\mathrm{C}^{\infty}$ coefficients, such that each derivative of each coefficient of $\eta$ has at most polynomial growth at infinity. Let $\mathrm{W}$ be the space of rapidly decreasing functions. Suppose that $W$ is contained in the domain of $H$. Let e $\in L_{2}\left(\mathbb{R}^{n}\right)$. For all $\phi \in \mathrm{W}$, let $\mathrm{B} \phi=\left(\Sigma_{\mathrm{i}=1}^{\mathrm{n}} \partial^{2} / \partial \mathrm{x}_{\mathrm{i}}^{2}-1\right)^{-\mathrm{M}}\left(\left(|\mathrm{x}|^{2}+1\right)^{-\gamma} \phi\right)$ for $\gamma>\mathrm{n} / 4$, where the inverse is taken in the space $W^{\prime}$, and where $M$ is such that $\left(|x|^{2}+1\right)^{-M}$ is in $L_{2}\left(\mathbb{R}^{n}\right)$. Then $B$ satisfies Assumption 1.2 and Estimate 1.3, with $\mathrm{f}=\left(|\mathrm{x}|^{2}+1\right)^{-\gamma}$, with $\mathrm{N}=0$, since $\mathrm{B}^{\prime} \theta=\left(|\mathrm{x}|^{2}+1\right)^{-\gamma}\left(\Sigma_{\mathrm{i}=1}^{\mathrm{n}} \partial^{2} / \partial \mathrm{x}_{\mathrm{i}}^{2}-1\right)^{-\mathrm{M}_{\theta} \text {, and since the hypotheses guarantee that }}$ 


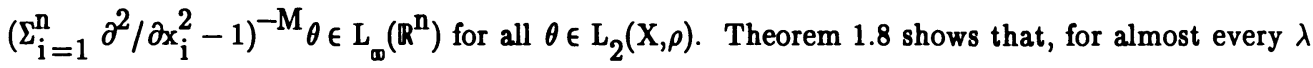
with respect to $\sigma_{e^{\prime}} F_{\lambda, e} \in W^{\prime}$ and $\left(|x|^{2}+1\right)^{-\gamma}\left(\Sigma_{i=1}^{n} \partial^{2} / \partial x_{i}^{2}-1\right)^{-N_{F}} F_{\lambda, e} \in L_{2}\left(\mathbb{R}^{\mathrm{n}}\right)$.

\section{APPROXIMATION}

In this section we study the main problem of the paper.

Assumption 3.1. Let $\mathrm{Y}$ be a subspace of $\mathrm{L}_{2}(\mathrm{X}, \rho)$ which is also a Banach space with norm \|\|$_{Y}$, and which has the properties that a) $W$ is a dense subspace of $Y, b$ ) there exists a constant $\mathrm{J}$ such that $\mathrm{J}\|\phi\|_{\mathrm{Y}} \geq\left\|\mathrm{B}^{-1} \phi\right\|_{2}$ for all $\phi \in \mathrm{W}$, and $\mathrm{c}$ ) the injection from $\mathrm{Y}$ into $\mathrm{L}_{2}(\mathrm{X}, \rho)$ is continuous. Let $\mathrm{Z}$ denote $\left\{\mathrm{F} \in \mathrm{W}^{\prime} \mid \mathrm{B}^{\prime} \mathrm{F} \in \mathrm{L}_{2}(\mathrm{X}, \rho)\right\}$; if $\mathrm{F} \in \mathrm{Z}$, let $\|\mathrm{F}\|_{\mathrm{Z}}=\left\|\mathrm{B}^{\prime} \mathrm{F}\right\|_{2}$. Let A denote the continuous extension of $\mathrm{B}^{-1}$ as an operator from $\mathrm{Y}$ into $\mathrm{L}_{2}(\mathrm{X}, \rho)$.

Remark: We now give the definition of diagonalization, and introduce a spectral measure which we denote by $\mu_{\mathrm{e}}$. Since the properties of $\mu_{\mathrm{e}}$ are very important for our theory, it is useful to note that by an elementary calculation it follows that the definition of $\mu_{\mathrm{e}}$ does not depend upon e, but only upon $\mathrm{S}_{\mathrm{e}}$. In other words, if $\mathrm{S}_{\mathrm{e}}=\mathrm{S}_{\mathrm{f}}$, then $\mu_{\mathrm{e}}=\mu_{\mathrm{f}}$. It should also be remarked that the following definition has been made quite detailed because it seems useful for later application to state the approximation properties we obtain completely.

Definition 3.2. Suppose the hypotheses of Assumption 3:1, Assumption 1.2 and Estimate 1.3 hold, and that $\mathrm{e} \in$ domain $\left(\mathrm{H}^{\mathrm{N}}\right)$. Let $\mu_{\mathrm{e}}$ be the positive measure on $\mathbb{R}$ defined by the relation $\mathrm{d} \mu_{\mathrm{e}}=\left\|\mathrm{B}^{\prime} \mathrm{F}_{\lambda, \mathrm{e}}\right\|_{2}^{2} \mathrm{~d} \sigma_{\mathrm{e}}$, where $\mathrm{F}_{\lambda, \mathrm{e}}$ is as in Theorem 1.8. Let $\mathrm{Q}=\mathrm{r}(\mathrm{H})$, where $\mathrm{r}$ is a bounded continuous function from the spectrum of the restriction of $H$ to $S_{e}$ into $C$. Let $\Delta$ be a Borel subset of $\mathbb{R}$. We say that $\mathrm{P}(\Delta) \mathrm{P}\left(\mathrm{S}_{\mathrm{e}}\right) \mathrm{Q}$ is diagonalizable in $\mathrm{B}(\mathrm{Y}, \mathrm{Z})$ with respect to $\mathrm{H}$ and $\mathrm{e}$ if $\mathrm{B}^{\prime} \mathrm{P}(\Delta) \mathrm{P}\left(\mathrm{S}_{\mathrm{e}}\right) \mathrm{Qg} \in \mathrm{L}_{2}(\mathrm{X}, \rho)$ for all $\mathrm{g} \in \mathrm{Y}$, and

a) for every $\epsilon>0$ there exists a positive integer $k$ and a finite disjoint family $\left\{\Delta_{i}\right\}_{i=1}^{k}$ of subsets of $\mathbb{R}$ such that $\mu_{\mathrm{e}}\left(\Delta_{\mathrm{i}}\right)$ is finite for every $i$ and such that there exists a set of real numbers $\left\{\lambda_{i}\right\}_{i=1}^{k}$ with $\lambda_{i} \in \Delta_{i} \cap \Delta$ and with the property that, injecting $\mathrm{P}(\Delta) \mathrm{P}\left(\mathrm{S}_{\mathrm{e}}\right) \mathrm{Q} \theta$ canonically into $\mathrm{W}^{\prime}$, $\left\|\left\{\mathrm{P}(\Delta) \mathrm{P}\left(\mathrm{S}_{\mathrm{e}}\right) \mathrm{Q}-\Sigma_{\mathrm{i}=1}^{\mathrm{k}} \mu_{\mathrm{e}}\left(\Delta_{\mathrm{i}} \cap \Delta\right) \overline{\mathrm{r}}\left(\lambda_{\mathrm{i}}\right) \mathrm{R}_{\lambda_{\mathrm{i}}}, \mathrm{e}\right\}(\theta)\right\|_{\mathrm{Z}} \leq \epsilon\|\theta\|_{\mathrm{Y}}$ for all $\theta \in \mathrm{Y}$, where

b) $\lambda_{i}$ is in the complement of the exceptional set of Theorem 1.8, so that in particular $F_{\lambda_{i}}, e$ is as in Theorem 1.8 and $B^{\prime} F_{\lambda_{i}, e} \in L_{2}(X, \rho)$ with $\left\|B^{\prime} F_{\lambda, e}\right\|_{2} \neq 0$, and where

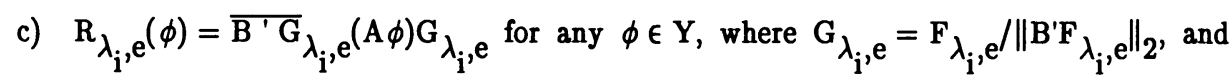
where $\overline{\mathrm{B}}$ ' $_{\lambda, \mathrm{e}}$ denotes the complex conjugate of the functional $\mathrm{B}^{\prime} \mathrm{G}_{\lambda, \mathrm{e}}$. The complex conjugate appears again, because we are working in $W^{\prime}$. Note that while the points $\lambda_{i}$ depend on $\Delta$, the number $\mathrm{k}$ and the sets $\Delta_{\mathrm{i}}$ do not; these depend only upon $\epsilon$. Note also that $\mathrm{R}_{\lambda_{\mathrm{i}}}, \mathrm{e}$ agrees with

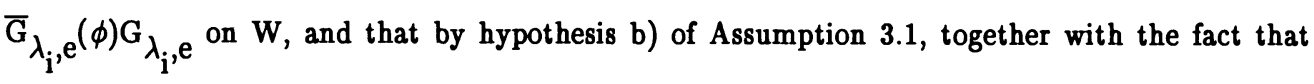
$B^{\prime} G_{\lambda_{i}, e} \in L_{2}(X, \rho)$, it follows that $R_{\lambda_{i}, e} \in B(Y, Z)$. 
Let $\mathrm{M}$ be a family of bounded continuous functions from the spectrum of the restriction of $\mathrm{H}$ to $\mathrm{S}_{\mathrm{e}}$ into $C$. Let $\mathrm{Q}_{\mathrm{M}}=\{\mathrm{r}(\mathrm{H}) \mid \mathrm{r} \in \mathrm{M}\}$. We say that $\mathrm{P}(\Delta) \mathrm{P}\left(\mathrm{S}_{\mathrm{e}}\right) \mathrm{Q}_{\mathrm{M}}$ is simultaneously diagonalizable in $\mathrm{B}(\mathrm{Y}, \mathrm{Z})$ with respect to $\mathrm{H}$ and $\mathrm{e}$ if for every $\epsilon>0$ there exist $\Delta_{\mathrm{i}}$ and $\lambda_{\mathrm{i}}$ as in a), b) and c) above such that $\left\|\left\{\mathrm{P}(\Delta) \mathrm{P}\left(\mathrm{S}_{\mathrm{e}}\right) \mathrm{Q}-\Sigma_{\mathrm{i}=1}^{\mathrm{k}} \mu_{\mathrm{e}}\left(\Delta_{\mathrm{i}} \cap \Delta\right) \overline{\mathrm{r}}\left(\lambda_{\mathrm{i}}\right) \mathrm{R}_{\lambda_{\mathrm{i}}}, \mathrm{e}\right\}(\theta)\right\|_{\mathrm{Z}} \leq \epsilon\|\theta\|_{\mathrm{Y}}$ for all $\theta \in \mathrm{Y}$ and all $\mathbf{r} \in \mathbf{M}$.

Theorem 3.3. Suppose that the hypotheses of Assumptions 3.1 and 1.2 hold, and that Estimate 1.3 holds, and $\mathrm{e} \in$ domain $\mathrm{H}^{\mathrm{N}}$. Let $\mathrm{G}_{\lambda, \mathrm{e}}=\mathrm{F}_{\lambda, \mathrm{e}} /\left\|\mathrm{B}^{\prime} \mathrm{F}_{\lambda, \mathrm{e}}\right\|_{2}$. Then Assertion i) below implies Assertion ii), which in turn implies Assertion iii).

i) $\Delta$ is a Borel subset of $\mathbb{R}$ such that $\left\{\mathrm{B}^{\prime} \mathrm{G}_{\lambda, \mathrm{e}} \mid \lambda \in \Delta\right\}$ is precompact in $\mathrm{L}_{2}(\mathrm{X}, \rho)$.

ii) $\int_{\Delta}\left\|\mathrm{B}^{\prime} \mathrm{F}_{\lambda, \mathrm{e}}\right\|_{2}^{2} \mathrm{~d} \sigma_{\mathrm{e}}(\lambda)<\infty$. (an elementary computation using the spectral theorem shows that $\int_{\Delta}\left\|\mathrm{B}^{\prime} \mathrm{F}_{\lambda, \mathrm{e}}\right\|^{2} \mathrm{~d} \sigma_{\mathrm{e}}=\int_{\Delta}\left\|\mathrm{B}^{\prime} \mathrm{F}_{\lambda, \mathrm{g}}\right\|^{2} \mathrm{~d} \sigma_{\mathrm{g}}$ if $\mathrm{g}$ is another cyclic vector for the subspace $\mathrm{S}_{\mathrm{e}}$.)

iii) Let $\mathrm{Q}=\mathrm{r}(\mathrm{H})$, where $\mathrm{r}$ is a bounded continuous function from the spectrum of the restriction of $\mathrm{H}$ to $\mathrm{S}_{\mathrm{e}}$ into $\mathrm{C}$. Then $\mathrm{P}(\Delta) \mathrm{P}\left(\mathrm{S}_{\mathrm{e}}\right) \mathrm{Q}$ is diagonalizable with respect to $\mathrm{H}$ and $\mathrm{e}$ in $\mathrm{B}(\mathrm{Y}, \mathrm{Z})$. If $\mathrm{M}$ is a set of bounded continuous functions from the spectrum of the restriction of $\mathrm{H}$ to $\mathrm{S}_{\mathrm{e}}$ into $\mathrm{C}$ which is uniformly bounded and equicontinuous on $\Delta$, then $\mathrm{P}(\Delta) \mathrm{P}\left(\mathrm{S}_{\mathrm{e}}\right) \mathrm{Q}_{\mathrm{M}}$ is simultaneously diagonalizable with respect to $\mathrm{H}$ and $\mathrm{e}$ in $\mathrm{B}(\mathrm{Y}, \mathrm{Z})$.

Proof: We note that Theorem 1.8 guarantees that $B^{\prime} F_{\lambda, e}$ is a measurable function from $\mathbb{R}$ into $\mathrm{L}_{2}(\mathrm{X}, \rho)$ with respect to $\sigma_{\mathrm{e}}$, in the sense that for every $\epsilon>0$ there exists a compact set $\mathrm{K}$ such that $\sigma_{\mathrm{e}}(\mathbb{R} \backslash \mathrm{K})<\epsilon$ and such that the restriction of $\mathrm{h}(\lambda)=\mathrm{B}^{\prime} \mathrm{F}_{\lambda, \mathrm{e}}$ to $\mathrm{K}$ is continuous from $\mathbb{R}$ into $\mathrm{L}_{2}(\mathrm{X}, \rho)$.

We show that Assertion i) implies Assertion ii). In fact, if Assertion i) holds, there exists a finite set $\left\{\lambda_{i}\right\}_{i=1}^{k}$ of points of $\Delta$ such that for all $\lambda \in \Delta$, there exists a $\lambda_{i}$ such that $\| B^{\prime} G_{\lambda, e^{-}}-B^{\prime} G_{\lambda_{i}}, e^{\|_{2}}<1 / 2$. Select a set $\left\{\phi_{i}\right\}_{i=1}^{k}$ of elements of $W$ such that $\left[B^{\prime} G_{\lambda_{i}, e^{e}}, \phi_{i}\right]>3 / 4$, and such that $\left\|\phi_{i}\right\|_{2}=1$. Let $\Delta=U_{i=1}^{k} \Delta_{i}$, where for $\lambda \in \Delta_{i},\left\|B^{\prime} G_{\lambda, e}-B^{\prime} G_{\lambda_{i}}, e\right\|_{2} \leq 1 / 2$. We may assume without loss of generality that the sets $\Delta_{i}$ are disjoint. It follows that for all $\lambda \in \Delta_{i}$, $\left|\left[\mathrm{B}^{\prime} \mathrm{G}_{\lambda, \mathrm{e}} \phi_{\mathrm{i}}\right]\right|>1 / 4$. Hence $\left|\left[\mathrm{B}^{\prime} \mathrm{F}_{\lambda, \mathrm{e}^{\mathrm{e}}}, \phi_{\mathrm{i}}\right]\right|>\left\|\mathrm{B}^{\prime} \mathrm{F}_{\lambda, \mathrm{e}}\right\|_{2} / 4$. But $\left[\phi_{\mathrm{i}}, \mathrm{B}^{\prime} \mathrm{F}_{\lambda, \mathrm{e}}\right]=\mathrm{U}_{\mathrm{e}}\left(\mathrm{B} \phi_{\mathrm{i}}\right)(\lambda)$. Hence $J_{\Delta}\left\|\mathrm{B}^{\prime} \mathrm{F}_{\lambda, \mathrm{e}}\right\|_{2}^{2} \mathrm{~d} \sigma_{\mathrm{e}}(\lambda) \leq 16 \Sigma_{\mathrm{i}=1}^{\mathrm{k}} \int_{\Delta_{\mathrm{i}}}\left|\mathrm{U}_{\mathrm{e}}\left(\mathrm{B} \phi_{\mathrm{i}}\right)\right|^{2} \mathrm{~d} \sigma_{\mathrm{e}}(\lambda)$. Since $\mathrm{B} \phi_{\mathrm{i}} \in \mathrm{W}$, the integral on the right is finite by the spectral theorem.

To complete the proof of the theorem, we need a lemma, which has some independent interest.

Lemma 3.4. Assume that Assertion ii) of Theorem 3.3 holds. Define $\mu_{\mathrm{e}}$ on $\Delta$ as above. Let $\gamma(\lambda)$ be defined on $\Delta$ by $\gamma(\lambda)=\mathrm{G}_{\lambda, \mathrm{e}}=\mathrm{F}_{\lambda, \mathrm{e}} /\left\|\mathrm{B}^{\prime} \mathrm{F}_{\lambda, \mathrm{e}}\right\|_{2}$ for all $\lambda$ in a subset $\Delta_{0}$ of $\Delta$ such that $\sigma_{\mathrm{e}}\left(\Delta \backslash \Delta_{0}\right)=0$. (This definition makes sense by Theorem 1.8). Then the following are true.

i) For any $\beta \in \mathrm{L}_{1}\left(\Delta, \mu_{\mathrm{e}}\right), \beta \gamma$ is a scalarwise integrable function from $\Delta$ into $\mathrm{W}^{\prime}$ with respect to $\mu_{\mathrm{e}}$, where by definition this means that for any $\phi \in \mathrm{W}, \beta \gamma(\phi) \in \mathrm{L}_{1}\left(\Delta, \mu_{\mathrm{e}}\right)$. In particular, this is true for $\beta \in \mathrm{L}_{\infty}\left(\mu_{\mathrm{e}}\right)$. 
ii) If $\Gamma(\beta)$ is defined for every $\beta \in \mathrm{L}_{1}\left(\mu_{\mathrm{e}}\right)$ and $\phi \in \mathrm{W}$ by

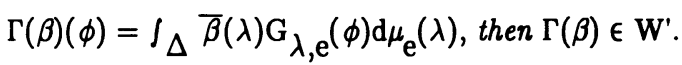

iii) For every $\beta \in \mathrm{L}_{1}\left(\Delta, \mu_{\mathrm{e}}\right), \beta \mathrm{B}^{\prime} \gamma$ is a scalarwise integrable function from $\Delta$ into $\mathrm{L}_{2}(\mathrm{X}, \rho)$ with respect to $\mu_{\mathrm{e}}$, where $\beta \mathrm{B}^{\prime} \gamma(\lambda)=\beta(\lambda) \mathrm{B}^{\prime} \mathrm{G}_{\lambda, \mathrm{e}}$.

iv) If $\Psi(\beta)$ is defined for every $\beta \in \mathrm{L}_{1}\left(\Delta, \mu_{\mathrm{e}}\right)$ and $\phi \in \mathrm{W}$ by $\Psi(\beta)(\phi)=\int_{\Delta} \bar{\beta}(\lambda) \mathrm{B}^{\prime} \mathrm{G}_{\lambda, \mathrm{e}}(\phi) \mathrm{d} \mu_{\mathrm{e}}(\lambda)$, then $\Psi(\beta) \in \mathrm{L}_{2}(\mathrm{X}, \rho)$, where $\mathrm{L}_{2}(\mathrm{X}, \rho)$ is identified with its canonical embedding into $\mathrm{W}^{\prime}$. Furthermore, $\|\Psi(\beta)\|_{2} \leq\|\beta\|_{1}$.

v) If $\beta \in \mathrm{L}_{1}\left(\Delta, \mu_{\mathrm{e}}\right), \mathrm{B}^{\prime} \Gamma(\beta)=\Psi(\beta)$.

vi) If $\theta \in \mathrm{L}_{2}(\mathrm{X}, \rho)$, and $\beta=\mathrm{U}_{\mathrm{e}}(\theta) / \| \mathrm{B}^{\prime} \mathrm{F}_{\lambda,} \mathrm{e}_{2}$, then $\beta \in \mathrm{L}_{1}\left(\Delta, \mu_{\mathrm{e}}\right)$ and $\Psi(\beta)=\mathrm{B}^{\prime} \mathrm{P}(\Delta) \mathrm{P}\left(\mathrm{S}_{\mathrm{e}}\right) \theta$, where $\mathrm{B}^{\prime} \mathrm{P}(\Delta) \mathrm{P}\left(\mathrm{S}_{\mathrm{e}}\right) \theta$ is identified with its canonical embedding into $\mathrm{W}^{\prime}$. In particular, $\mathrm{B}^{\prime} \mathrm{P}(\Delta) \mathrm{P}\left(\mathrm{S}_{\mathrm{e}}\right) \theta \in \mathrm{L}_{2}(\mathrm{X}, \rho)$.

Proof: Part iii) follows from the finiteness of the measure $\mu_{\mathrm{e}}$, together with the boundedness of $B^{\prime} G_{\lambda, e}$ in $L_{2}(X, \rho)$. Since $B$ is $1-1$ and onto, part $\left.i\right)$ follows as well. Since $\mu_{e}$ is finite on $\Delta, \mathrm{L}_{\infty}\left(\Delta, \mu_{\mathrm{e}}\right) \subset \mathrm{L}_{1}\left(\mu_{\mathrm{e}}\right)$. We show that $\Psi(\beta) \in \mathrm{W}^{\prime}$. By Theorem 1.8, we know that $\Psi$ is a measurable function from $\Delta$ into $\mathrm{L}_{2}(\mathrm{X}, \rho)$. Furthermore, $|\Psi(\beta)(\phi)| \leq\|\beta\|_{1}\|\phi\|_{2}$, so we see that $\Psi(\beta)$ agrees on $\mathrm{W}$ with a unique element of $\mathrm{L}_{2}(\mathrm{X}, \rho)$ defined via the Riesz representation theorem for Hilbert spaces by the relationship $\Psi(\beta)(\theta)=\int_{\Delta} \bar{\beta}(\lambda)\left[\theta, \mathrm{B}^{\prime} \mathrm{G}_{\lambda,} \mathrm{e}^{\mathrm{d}} \mu_{\mathrm{e}}(\lambda)\right.$ for all $\theta \in \mathrm{L}_{2}(\mathrm{X}, \rho)$. Part iv) is proved. Since $\left(B^{\prime}\right)^{-1}$ is a continuous linear mapping from $L_{2}(X, \rho)$ into $W^{\prime}$, (because the injection of $L_{2}(X, \rho)$ into $W^{\prime}$ is continuous, and $\left(B^{\prime}\right)^{-1}$ is a continuous linear transformation in $\left.W^{\prime}\right)$, where we once again identify $\mathrm{L}_{2}(\mathrm{X}, \rho)$ with its canonical embedding into $\mathrm{W}^{\prime}$, we may use Theorem 8.14.5, p. 562, [4] to obtain part ii) and part v). We prove part vi).

If $\phi \in \mathrm{W}$, it follows from part iv) that $[\phi, \Psi(\beta)]=\int_{\Delta} \bar{\beta}(\lambda)\left[\phi, \mathrm{B}^{\prime} \mathrm{G}_{\lambda, \mathrm{e}}\right] \mathrm{d} \mu_{\mathrm{e}}(\lambda)$. Also,

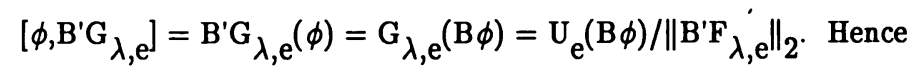

$\int_{\Delta} \bar{\beta}(\lambda)\left[\phi, \mathrm{B}^{\prime} \mathrm{G}_{\lambda, \mathrm{e}^{\mathrm{e}}}\right] \mathrm{d} \mu_{\mathrm{e}}(\lambda)=\int_{\Delta}\left(\mathrm{U}_{\mathrm{e}}(\mathrm{B} \phi) /\left\|\mathrm{B}^{\prime} \mathrm{F}_{\lambda, \mathrm{e}}\right\|_{2}\right)\left\{\left(\overline{\mathrm{U}_{\mathrm{e}}} \bar{\theta}\right) /\left\|\mathrm{B}^{\prime} \mathrm{G}_{\lambda, \mathrm{e}}\right\|_{2}\right\} \mathrm{d} \mu_{\mathrm{e}}(\lambda)$. But

$\mathrm{d} \mu_{\mathrm{e}}=\left\|\mathrm{B}^{\prime} \mathrm{F}_{\lambda, \mathrm{e}}\right\|^{2} \mathrm{~d} \sigma_{\mathrm{e}}$, so the integral on the right becomes $J_{\Delta}\left(\mathrm{U}_{\mathrm{e}}(\mathrm{B} \phi)\right)\left(\overline{\mathrm{U}_{\mathrm{e}}}{ }^{\theta}\right) \mathrm{d} \sigma_{\mathrm{e}}(\lambda)$, which equals $[\mathrm{B} \phi, \theta]$ by the spectral theorem; this theorem also guarantees that the integrand is in $\mathrm{L}_{1}\left(\sigma_{\mathrm{e}}\right)$, and also that $\beta \in \mathrm{L}_{2}\left(\mu_{\mathrm{e}}\right)$. Since $\mu_{\mathrm{e}}$ is finite on $\Delta$, it follows that $\beta \in \mathrm{L}_{1}\left(\Delta, \mu_{\mathrm{e}}\right)$. But if $\alpha=\mathrm{P}(\Delta) \mathrm{P}\left(\mathrm{S}_{\mathrm{e}}\right) \theta,[\mathrm{B} \phi, \alpha]=\alpha(\mathrm{B} \phi)=\mathrm{B}^{\prime} \alpha(\phi)$, again embedding $\mathrm{L}_{2}(\mathrm{X}, \rho)$ canonically into $\mathrm{W}^{\prime}$. We have therefore seen that $\mathrm{B}^{\prime} \alpha=\Psi(\beta)$, as we desired to show. The lemma is proved.

We now show that Assertion ii) implies Assertion iii) of the theorem. For any positive real number $\delta$, select a compact subset $\mathrm{K}$ of $\Delta$ such that $\mathrm{h}$ is a continuous function from $\mathrm{K}$ into $\mathrm{L}_{2}(\mathrm{X}, \rho)$, and such that $\mu_{\mathrm{e}}(\Delta \mid \mathrm{K})<\delta$. Note that $\{\mathrm{h}(\lambda) \mid \lambda \in \mathrm{K}\}$ is a compact subset of $\mathrm{L}_{2}(\mathrm{X}, \rho)$. Let $\{\nu(\mathrm{i})\}$ be a finite open cover of this set where each $\nu(\mathrm{i})$ has diameter less than $\delta$. Let 
$\alpha(\mathrm{j})=\mathrm{h}^{-1}(\nu(\mathrm{j}))$. Select a finite relatively open cover $\{\aleph(\mathrm{n})\}$ of $\mathrm{K}$ which is subordinate to $\{\alpha(\mathrm{j})\}$, and which has the property that $|\mathrm{r}(\lambda)-\mathrm{r}(\beta)|<\delta$ for all $\lambda$ and $\beta$ in $\aleph(\mathrm{n})$. Let $\left.\zeta(\mathrm{j})=\aleph(\mathrm{j}) \backslash U_{\mathrm{i}<\mathrm{j}} \mathrm{N}^{\mathrm{N}} \mathrm{i}\right)$. Let $b_{j}=\mu_{e}(\zeta(j))$. Select one $\lambda_{j}$ from each set $\zeta(j)$ and let $Q_{0}=\Sigma_{j=1}^{k} \bar{r}\left(\lambda_{j}\right) b_{j} R_{\lambda_{j}}, e^{\prime}$ using the notation of Definition 3.2. Note that for $\phi \in W$, since $\left\|B^{\prime} G_{\lambda, e}\right\|_{2}=1$,

$$
\left|G_{\lambda, e^{(}}(\phi)\right|=\left|B^{\prime} G_{\lambda, e}\left(B^{-1} \phi\right)\right|=\left|\left[B^{\prime} G_{\lambda, e} B^{-1} \phi\right]\right| \leq\left\|B^{-1} \phi\right\|_{2}\left\|B^{\prime} G_{\lambda, e}\right\|_{2} \leq J\|\phi\|_{Y}
$$

Using Lemma 3.4, parts iv), v) and vi), together with Proposition 8.14.6, p. 562, Edwards [4], used on the space $L_{2}(X, \rho)$, we see that for any $\phi \in W$ such that $\|\phi\|_{Y}=1$, the function mapping $\lambda$ to $\overline{\mathrm{r}}(\lambda) \overline{\mathrm{G}}_{\lambda, \mathrm{e}}(\phi) \mathrm{G}_{\lambda, \mathrm{e}}$ is a scalarwise integrable function from $\mathbb{R}$ into $\mathrm{W}^{\prime}$ with respect to $\sigma_{\mathrm{e}}$, and

$$
\begin{aligned}
& \left\|\mathrm{B}^{\prime}\left\{\mathrm{P}\left(\mathrm{S}_{\mathrm{e}}\right) \mathrm{P}(\Delta) \mathrm{Q} \phi-\mathrm{Q}_{0} \phi\right\}\right\|_{2}= \\
& \| B^{\prime}\left\{\Sigma _ { j } J _ { \zeta ( j ) } \left(\bar{r}(\lambda) \bar{G}_{\left.\lambda, e^{(}\right)}(\phi) G_{\lambda, e^{-}}-\bar{r}\left(\lambda_{j}\right) \bar{G}_{\lambda_{j}} e^{\left.(\phi) G_{\lambda}\right) d \mu_{e}(\lambda)+}\right.\right.
\end{aligned}
$$

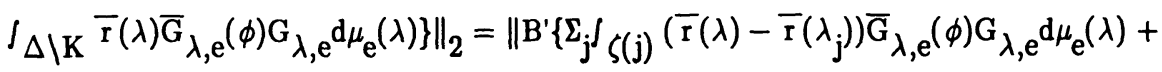

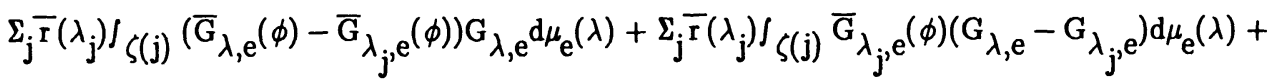

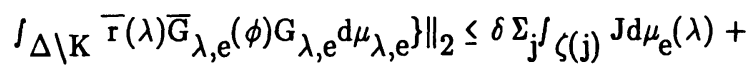

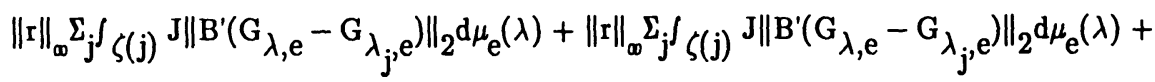

$$
\begin{aligned}
& \|\mathrm{r}\|_{\infty} \mathrm{J} \int_{\Delta \backslash \mathrm{K}} \mathrm{d} \mu_{\mathrm{e}}(\lambda) \leq \delta \mathrm{J} \mu_{\mathrm{e}}(\Delta)+\mathrm{J}\|\mathrm{r}\|_{\infty} \delta \mu_{\mathrm{e}}(\Delta)+\mathrm{J}\|\mathrm{r}\|_{\infty} \delta \mu_{\mathrm{e}}(\Delta)+\mathrm{J}\|\mathrm{r}\|_{\infty} \delta .
\end{aligned}
$$

We have seen that for any $\phi \in \mathrm{W}$ such that $\|\phi\|_{\mathrm{Y}}=1$,

$$
\left\|\mathrm{B}^{\prime}\left\{\mathrm{P}\left(\mathrm{S}_{\mathrm{e}}\right) \mathrm{P}(\Delta) \mathrm{Q} \phi-\mathrm{Q}_{0} \phi\right\}\right\|_{2} \leq \delta\left\{\mathrm{J} \mu_{\mathrm{e}}(\Delta)\left(1+2\|\mathrm{r}\|_{\infty}\right)+\mathrm{J}\|\mathrm{r}\|_{\infty}\right\} .
$$

We must extend this estimate to all of $Y$.

We examine the operators $\mathrm{B}^{\prime} \mathrm{P}\left(\mathrm{S}_{\mathrm{e}}\right) \mathrm{P}(\Delta) \mathrm{Q}$ and $\mathrm{B}^{\prime} \mathrm{P}\left(\mathrm{S}_{\mathrm{e}}\right) \mathrm{P}(\Delta) \mathrm{Q}_{0}$. Note that by Lemma 3.4, for any $\theta$ in $\mathrm{L}_{2}(\mathrm{X}, \rho), \Psi(\beta)=\mathrm{B}^{\prime} \mathrm{P}(\Delta) \mathrm{P}\left(\mathrm{S}_{\mathrm{e}}\right) \theta$, where $\beta=\mathrm{U}_{\mathrm{e}}(\theta) /\left\|\mathrm{B}^{\prime} \mathrm{F}_{\lambda, \mathrm{e}}\right\|_{2}$. Again by Lemma 3.4, $\Psi(\beta) \in \mathrm{L}_{2}(\mathrm{X}, \rho)$, and $\|\Psi(\beta)\|_{2} \leq\|\beta\|_{1}$. However, since $\mu$ is finite on $\Delta$, it follows that $\|\Psi(\beta)\|_{2} \leq\left(\mu_{\mathrm{e}}(\Delta)\right)^{1 / 2}\|\beta\|_{2}$ by the Schwartz inequality. But the spectral theorem, and the definition of $\mu_{\mathrm{e}}$, show that $\left.\|\aleph(\Delta) \beta\|_{2, \mu_{\mathrm{e}}}=\| \mathrm{P}(\Delta)\right)\left(\mathrm{S}_{\mathrm{e}}\right) \theta \|_{2, \rho}$. If $\theta=\mathrm{Q} \phi$, with $\phi \in \mathrm{Y}$, and if we recall that by Assumption 3.1 the identity mapping $\mathrm{E}_{\mathrm{Y}}$ from $\mathrm{Y}$ into $\mathrm{L}_{2}(\mathrm{X}, \rho)$ is continuous, we see from part vi) of Lemma 3.4 that $\mathrm{B}^{\prime} \mathrm{P}(\Delta) \mathrm{P}\left(\mathrm{S}_{\mathrm{e}}\right) \mathrm{Q}$ is a continuous mapping from $\mathrm{Y}$ into $\mathrm{L}_{2}(\mathrm{X}, \rho)$.

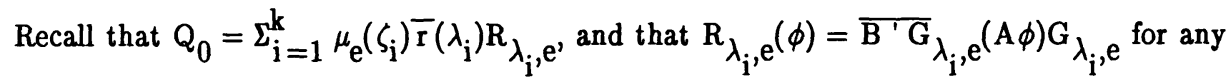
$\phi \in \mathrm{Y}$, where $\mathrm{A}$ is the continuous extension of $\mathrm{B}^{-1}$ to $\mathrm{Y}$, as an operator from $\mathrm{Y}$ into $\mathrm{L}_{2}(\mathrm{X}, \rho)$. 
Observe that we have selected $\lambda_{i}$ in such a way that $B^{\prime} F_{\lambda_{i}, e} \in L_{2}(X, \rho)$. Furthermore, $\mathrm{B}^{\prime} G_{\lambda_{\mathrm{i}, \mathrm{e}}}(\mathrm{A} \phi)=\left[\mathrm{A} \phi, \mathrm{B}^{\prime} \mathrm{G}_{\lambda_{\mathrm{i}}, \mathrm{e}}\right]$. Since $\mathrm{A}$ is continuous from $\mathrm{Y}$ into $\mathrm{L}_{2}(\mathrm{X}, \rho)$, it follows that $\mathrm{Q}_{0}$ is a bounded linear transformation from $\mathrm{Y}$ into $\mathrm{L}_{2}(\mathrm{X}, \rho)$. Assertion iii) of Theorem 3.3 follows by continuity, since $\mathrm{W}$ is dense in $\mathrm{Y}$. The theorem is proved.

\section{A SIMPLE EXAMPLE}

In this section, to place the results in perspective, we study the situation of a second order ordinary differential operator in $\mathrm{L}_{2}([0, \infty))$. We show that in considerable generality in the second-order ode case, there exist unbounded sets $\Delta$ and natural spaces $\mathrm{Y}$ and $\mathrm{Z}$ such that $\mathrm{P}(\Delta)$ is diagonalizable (and therefore compact) in $\mathrm{B}(\mathrm{Y}, \mathrm{Z})$, but such that the injection from $\mathrm{Y}$ into $\mathrm{Z}$ is not compact. Hence in this situation the compactness of $P(\Delta)$ is a consequence, rather than a hypothesis, for our theory. For p identically 1 and $x q(x) \in L_{1}$, sets $\Delta$ have this property for the $\mathrm{Y}$ and $Z$ of this section if $1 / \sqrt{\lambda} \in L_{1}(\Delta \cap(0, \infty))$, as very recent results of $D$. B. Hinton and the author [4] show, using the results of this paper. A number of lemmas are proved in this section, which seem fairly obvious, such as the existence of a cyclic vector. The proofs and statements are included because the author is unable to find them in the literature, in the continuous spectrum case.

Definition 4.1. Let $\tau$ be given by $\tau \phi=-\left(\mathrm{p} \phi^{\prime}\right)^{\prime}+\mathrm{q} \phi$ for all sufficiently differentiable $\phi$ on $[0, \infty)$, where $p$ and $q$ are $C^{\infty}$ functions from $[0, \infty)$ into $\mathbb{R}$, and where $p$ is positive and bounded away from 0 and $q$ is bounded below. Let $\mathrm{H}$ be the Friedrichs extension in $\mathrm{L}_{2}[0, \infty)$ of the restriction of $\tau$ to $C_{0}^{\infty}(0, \infty)$. It is clear that any element $\phi$ of the domain of $H$ is actually in $L_{\infty}[0, \infty)$, since the hypotheses guarantee that for such a $\phi, \phi^{\prime}$ is in $\mathrm{L}_{2}=\mathrm{L}_{2}[0, \infty)$. Throughout this section let $\omega$ be any positive bounded $C^{\infty}$ element of $L_{2}$; let $Z_{\omega}$ denote $\left\{F: \omega F \in L_{2}\right\}$, and $Y_{\omega}$ denote $\left\{\phi: \phi / \omega \in \mathrm{L}_{2}\right\}$.

Remark 4.2. If $\mathrm{W}=\mathrm{C}_{0}^{\infty}(0, \infty)$ and $\mathrm{B}$ is the operator of multiplication by $\omega$, then Assumption 1.2, Estimate 1.3, and Assumption 3.1 hold for the above $H$, with $f=\omega, Y=Y_{\omega}$ and $\mathrm{Z}=\mathrm{Z}_{\omega}$. In particular, Theorem 3.3 and Lemma 3.4 hold. We show that there is an element $\mathrm{e}$ of Y such that $\mathrm{S}_{\mathrm{e}}=\mathrm{L}_{2}$. This requires another functional analytic result.

Theorem 4.3. Suppose that Assumptions 1.2 and 9.1 and Estimate 1.9 hold. Then for any $\mathrm{e} \in \mathrm{D}\left(\mathrm{H}^{\mathrm{N}}\right)$ and almost every $\lambda$ with respect to $\sigma_{\mathrm{e}}$, there exists a decreasing tower $\left\{\Delta_{\mathrm{n}}\right\}$ of compact sets with the following properties:

a) $\Delta_{\mathrm{n}} \mathrm{c}[\lambda-1 / \mathrm{n}, \lambda+1 / \mathrm{n}]$

b) $\sigma_{\mathrm{e}}\left(\Delta_{\mathrm{n}}\right)>0$;

c) the mapping $\lambda \rightarrow \mathrm{G}_{\lambda, \mathrm{e}}$ is continuous from $\Delta_{\mathrm{n}}$ into $\mathrm{Z}$;

d) $\mathrm{P}\left(\Delta_{\mathrm{n}}\right) \mathrm{e} / \mu_{\mathrm{e}}\left(\Delta_{\mathrm{n}}\right)$ converges in $\mathrm{Z}$ to $\mathrm{G}_{\lambda, \mathrm{e}} /\left\|\mathrm{B}^{\prime} \mathrm{F}_{\lambda, \mathrm{e}_{2}}\right\|_{2}$

Proof: We showed in Theorem 1.8 that the mapping $\gamma: \lambda \rightarrow \mathrm{G}_{\lambda, \mathrm{e}}$ is a measurable function from $\mathbb{R}$ into $Z$. Hence there exists a tower of compact sets $K_{j}$ such that $\sigma_{e}\left(\mathbb{R} \mid U_{j=1}^{\infty} K_{j}\right)=0$, and such that the restriction of $\gamma$ to $\mathrm{K}_{\mathrm{j}}$ is continuous from $\mathrm{K}_{\mathrm{j}}$ into $\mathrm{Z}$. Let 
$\Delta_{\mathbf{n}}=[\lambda-1 / \mathbf{n}, \lambda+1 / \mathrm{n}] \cap \mathrm{K}_{\mathrm{j}}$ If $\sigma_{\mathrm{e}}\left(\Delta_{\mathrm{n}}\right) \neq 0$ for all $\mathrm{n}$, and $\lambda \in \Delta_{\mathbf{n}}$ for all $\mathrm{n}$, then letting $\beta_{\mathbf{n}}$ denote the characteristic function of $\Delta_{n}$, Lemma 3.4, part vi) guarantees that

$\Gamma\left(\beta_{\mathrm{n}}(\mathrm{s}) /\left\|\mathrm{B}^{\prime} \mathrm{F}_{\mathrm{s}, \mathrm{e}}\right\|_{2}\right)=\mathrm{P}\left(\Delta_{\mathrm{n}}\right) \mathrm{e}$, since $\mathrm{U}_{\mathrm{e}}(\mathrm{e}) \equiv 1$ and since $\left\|\mathrm{B}^{\prime} \mathrm{F}_{\lambda, \mathrm{e}}\right\|_{2}$ is bounded away from 0 on $\Delta_{n}$, because it is continuous and non-vanishing. But it is clear from vi) of Lemma 3.4 together with the continuity of $\gamma$ that conclusion d) of the theorem holds. However, the set of all $\lambda$ such that for every set $K_{j}$ containing $\lambda$ there is an $n$ such that $\sigma_{e}\left([\lambda-1 / n, \lambda+1 / n] \cap K_{j}\right)=0$ is a set of measure 0 with respect to $\sigma_{\mathrm{e}}$. The theorem is proved.

Theorem 4.4. Let $\mathrm{H}$ be as in Definition 4.1. Let $\mathrm{W}$ and $\mathrm{B}$ satisfy the hypotheses of Assumptions 1.2 and 3.1 and Estimate 1.9; assume further that $\mathrm{C}_{0}^{\infty}(0, \infty)$ is algebraically and topologically contained in $\mathrm{W}$, where $\mathrm{C}_{0}^{\infty}(0, \infty)$ is given the usual inductive limit topology. (Recall that all $\phi \in \mathrm{C}_{0}^{\infty}(0, \infty)$ vanish in a neighborhood of 0$)$. Suppose e $\in$ Domain $(\mathrm{H})$. Then $\mathrm{F}_{\lambda, \mathrm{e}} \in \mathrm{C}^{\infty}[0, \infty)$ and $\tau \mathrm{F}_{\lambda, \mathrm{e}}=\lambda \mathrm{F}_{\lambda, \mathrm{e}}$ for every $\lambda$ in the set $\zeta$ of Theorem 1.8, and $\mathrm{F}_{\lambda, \mathrm{e}}(0)=0$ for almost every $\lambda$ in $\zeta$ with respect to $\sigma_{\mathrm{e}}$.

Proof: Let $\lambda \in \zeta$. Then $\mathrm{H}^{\prime} \mathrm{F}_{\lambda, \mathrm{e}}=\lambda \mathrm{F}_{\lambda, \mathrm{e}^{\prime}}$ by Theorem 1.8. Hence, for any $\theta$ in $\mathrm{W}$, $\mathrm{F}_{\lambda, \mathrm{e}}(\tau \theta)=\lambda \mathrm{F}_{\lambda, \mathrm{e}}(\theta)$. In particular this is true for $\theta$ in $\mathrm{C}_{0}^{\infty}(1, \infty)$, so that $\tau \mathrm{F}_{\lambda, \mathrm{e}}=\lambda \mathrm{F}_{\lambda, \mathrm{e}}$ in the space of distributions. But any distributional solution to $\tau \mathrm{F}_{\lambda, \mathrm{e}}=\lambda \mathrm{F}_{\lambda, \mathrm{e}}$ is in $\mathrm{C}^{\infty}[0, \infty)$. We need only show that $\mathrm{F}_{\lambda, \mathrm{e}}(0)=0$ for almost every $\lambda$ with respect to $\sigma_{\mathrm{e}}$. For this, we may choose the spaces $\mathrm{W}$ and $\mathrm{Y}$ and the map $\mathrm{B}$ any way we wish. Choose $\mathrm{W}$ to be $C_{0}^{\infty}(0, \infty)$ and $\mathrm{B}$ to be multiplication by $\omega$, with $\mathrm{f}=\omega$. By Theorem 4.2 we may construct a sequence $\Delta_{\mathrm{n}}$ of compact sets contained in $[\lambda+1 / n, \lambda-1 / n]$ for almost every $\lambda$ such that $\xi_{n}=P\left(\Delta_{n} e\right) / \mu_{e}\left(\Delta_{n}\right)$ converges in $\mathrm{Z}_{\delta}$ to $\mathrm{G}_{\lambda, \mathrm{e}} /\left\|\omega \mathrm{F}_{\lambda, \mathrm{e}}\right\|_{2}$. But since $\Delta_{\mathrm{n}} \mathrm{C}[\lambda-1 / \mathrm{n}, \lambda+1 / \mathrm{n}]$, it follows that $\tau \xi_{\mathrm{n}}$ converges in $\mathrm{Z}_{\delta}$ to $\lambda G_{\lambda, \mathrm{e}} /\left\|\omega \mathrm{F}_{\lambda, \mathrm{e}}\right\|_{2}$. Hence, if $\nu_{\mathrm{n}}=\omega \xi_{\mathrm{n}}$, we see that $\nu_{\mathrm{n}}$ converges in $\mathrm{L}_{2}$ to $\omega \mathrm{G}_{\lambda, \mathrm{e}} /\left\|\omega \mathrm{F}_{\lambda, \mathrm{e}}\right\|_{2}$ and $\omega \tau\left(\nu_{\mathrm{n}} / \omega\right)$ converges in $\mathrm{L}_{2}$ to $\lambda \omega G_{\lambda, \mathrm{e}} /\left\|\omega \mathrm{F}_{\lambda, \mathrm{e}}\right\|_{2}$. Writing out the terms of the differential expression $\beta$ such that $\beta \theta=\omega \tau(\theta / \omega)$, we see that $\nu_{\mathrm{n}}$ converges uniformly on compacta to $\omega G_{\lambda, \mathrm{e}} /\left\|\omega \mathrm{F}_{\lambda, \mathrm{e}}\right\|_{2}$; in particular this is true in a neighborhood of 0 . It follows immediately that $\mathrm{G}_{\lambda, \mathrm{e}}(0)=0$, as we desired to prove.

Theorem 4.5. Let $\mathrm{H}$ be as in Definition 4.1. Then there exists an $\mathrm{e}$ in the domain of $\mathrm{H}$ such that $\mathrm{S}_{\mathrm{e}}=\mathrm{L}_{2}$.

Proof: Choose $\mathrm{W}=\mathrm{C}_{0}^{\infty}(0, \infty)$, and choose B in Estimate 1.3 to be multiplication by $\omega$ and $\mathrm{f}=\omega$. Choose $\mathrm{Z}=\mathrm{Z}_{\omega}$. From the spectral theorem, there exists an $\mathrm{e} \in \mathrm{L}_{2}$ such that for any $g \in \mathrm{L}_{2}, \sigma_{\mathrm{g}}$ is absolutely continuous with respect to $\sigma_{\mathrm{e}}$. Without loss of generality, e may be taken in the domain of $\mathrm{H}$. We show that $\mathrm{S}_{\mathrm{e}}=\mathrm{L}_{2}$. If not, there exists a non-trivial element $\mathrm{g}$ of the domain of $\mathrm{H}$ such that $\mathrm{S}_{\mathrm{g}} \perp \mathrm{S}_{\mathrm{e}}$. By the previous theorem, for almost every $\lambda$ with respect to $\mathrm{S}_{\mathrm{e}^{\prime}}$

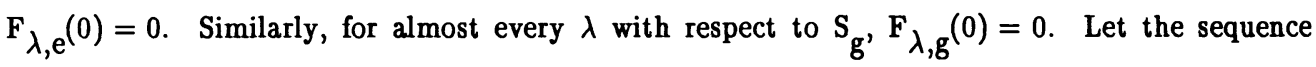
$\phi_{\mathrm{n}} \in \mathrm{C}_{0}^{\infty}(0, \infty)$ be such that $\left\|\phi_{\mathrm{n}}\right\|_{2}=1$ and also such that $\phi_{\mathrm{n}}$ converges to $\mathrm{g}$ in $\mathrm{L}_{2}$. Selecting a 
subsequence, which we also denote by $\phi_{n}$, we see that $\mathrm{U}_{\mathrm{e}}\left(\phi_{\mathrm{n}}\right)(\lambda)$ converges to 0 for almost every $\lambda$ with respect to $\sigma_{\mathrm{e}}$ and $\mathrm{U}_{\mathrm{g}}\left(\phi_{\mathrm{n}}\right)(\lambda)$ converges to 1 for almost every $\lambda$ with respect to $\sigma_{\mathrm{g}}$. Hence, since $\sigma_{\mathrm{g}}$ is absolutely continuous with respect to $\sigma_{\mathrm{e}}$, we see that for almost every $\lambda$ with respect to $\sigma_{\mathrm{g}}, \mathrm{F}_{\lambda, \mathrm{e}}\left(\phi_{\mathrm{n}}\right)$ converges to 0 and $\mathrm{F}_{\lambda, \mathrm{g}}\left(\phi_{\mathrm{n}}\right)$ converges to 1 . But $\mathrm{F}_{\lambda, \mathrm{e}}$ must be a multiple of $\mathrm{F}_{\lambda, \mathrm{g}}$ for almost every $\lambda$ with respect to $\sigma_{\mathbf{g}}$, since both vanish at 0 . This is a contradiction; the theorem is proved.

Remark: We now prove a negative result, showing that some hypotheses are necessary in order to diagonalize on a Borel set $\Delta$. The first operator one might wish to diagonalize is the identity operator; this gives a discrete approximation to an inverse Fourier transform.

Theorem 4.6. Let $\mathrm{H}$ be as in Definition 4.1. Suppose that $\mathrm{P}(\Delta)$ is diagonalizable in $\mathrm{B}\left(\mathrm{Y}_{\omega}, \mathrm{Z}_{\omega}\right)$ with respect to $\mathrm{H}$. Then $\mathrm{P}(\Delta)$ is a compact operator from $\mathrm{Y}_{\omega}$ into $\mathrm{Z}_{\omega}$. In particular, the identity operator $\mathrm{P}(\mathbb{R})$ is not diagonalizable in $\mathrm{B}\left(\mathrm{Y}_{\omega}, \mathrm{Z}_{\omega}\right)$ with respect to $\mathrm{H}$.

Remark: Since the embedding of $\mathrm{Y}_{\omega}$ into $\mathrm{L}_{2}$ is continuous, as is the embedding of $\mathrm{L}_{2}$ into $\mathrm{Z}_{\omega}$, it follows that $\mathrm{P}(\Delta)$ is in $\mathrm{B}\left(\mathrm{Y}_{\omega}, \mathrm{Z}_{\omega}\right)$. Since the previous theorem showed that there exists an element $\mathrm{e}$ of the domain of $\mathrm{H}$ such that $\mathrm{S}_{\mathbf{e}}=\mathrm{L}_{2}$, we do not need to consider $\mathrm{P}\left(\mathrm{S}_{\mathrm{e}}\right)$.

Proof: Operators with finite dimensional range are compact, as are limits in operator norm of such operators. The first conclusion is therefore immediate. The embedding of $Y_{\omega}$ into $Z_{\omega}$ is clearly not compact, since on the interval $[0,1]$ the norm of $Y_{\omega}$ is equivalent to that of $Z_{\omega}$ and to the norm of $\mathrm{L}_{2}([0,1])$.

Theorem 4.7. Let $\mathrm{H}$ be as in Definition 4.1; let $\mathrm{e}$ be any element of the domain of $\mathrm{H}$ such that $\mathrm{S}_{\mathrm{e}}=\mathrm{L}_{2}$. Then

a) for any bounded continuous function $\mathrm{r}$ : spectrum $(\mathrm{H}) \rightarrow \mathrm{C}$, and any bounded Borel set $\Delta$, $\mathrm{P}(\Delta) \mathrm{r}(\mathrm{H})$ is diagonalizable in $\mathrm{B}\left(\mathrm{Y}_{\omega}, \mathrm{Z}_{\omega}\right)$ with respect to $\mathrm{H}$;

b) if the essential spectrum of $\mathrm{H}$ is not a bounded set, there exist subsets $\Delta$ of the spectrum of $\mathrm{H}$ such that $\mu_{\mathrm{e}}(\Delta)$ is finite (where $\mathrm{d} \mu_{\mathrm{e}}=\left\|\mathrm{F}_{\lambda,}\right\|_{\mathrm{Z}_{\omega}}^{2} \mathrm{~d} \sigma_{\mathrm{e}}$ ), and such that for all $\mathrm{N}$, $\sigma_{\mathrm{e}}(\Delta \backslash[-\mathrm{N}, \mathrm{N}])>0$. In particular, for such $\Delta$ and any bounded continuous function $\mathrm{r}(\mathrm{H})$ of $\mathrm{H}$, $\mathrm{P}(\Delta) \mathrm{r}(\mathrm{H})$ is diagonalizable with respect to $\mathrm{H}$ in $\mathrm{B}\left(\mathrm{Y}_{\omega}, \mathrm{Z}_{\omega}\right)$, although $\Delta$ is not an essentially bounded set with respect to $\sigma_{\mathrm{e}}$.

Remark: The first conclusion of the theorem does not state that $\mu_{\mathrm{e}}$ is finite on bounded sets. This question is a difficult one, which we do not address here.

Proof: The first assertion will be proved in more generality in Theorem 5.5 of the next section. If the second assertion of the theorem is false, then the finiteness of $\mu_{\mathrm{e}}(\Delta)$ implies that for some $N, \sigma_{e}(\Delta \backslash[-N, N])=0$. This implies that if $a_{i}=\mu_{e}([N+i, N+i+1)), \quad\left\{a_{i}: a_{i}>0\right\}$ is bounded away from 0 in $(0, \infty)$. Since the operator $H$ is unbounded, if $\Gamma=\left\{i: a_{i}>0\right\}$, then $\Gamma$ is infinite. It is also clear that if $b_{i}=\underset{J c[N+i, N+i+1)}{g l b}\left\{\mu_{e}(J): \mu_{e}(J)>0\right\}$, then $\left\{b_{j}: j \in \Gamma\right\}$ is also bounded away from 0 . Suppose $\lambda>N$. Either $\lambda$ is in the exceptional set of Theorem 4.3, or there 
exists a decreasing tower $\Delta_{n}$ of Borel sets such that $\lambda=n_{n=1}^{\infty} \Delta_{n}$, and such that $\mu_{e}\left(\Delta_{n}\right)$ is finite and positive for each $\mathrm{n}$. Since by hypothesis, $\mu_{\mathrm{e}}\left(\Delta_{\mathrm{n}}\right)$ does not approach 0 , this implies that $\lambda$ is a point mass. It follows that there exists a countable set $\left\{\lambda_{n}\right\}$ of points of the spectrum of $H$ such that $\mu_{\mathrm{e}}\left([\mathrm{N}, \boldsymbol{\infty}) \backslash\left\{\lambda_{\mathrm{n}}\right\}\right)=0$. Hence, the same assertion is true for $\sigma_{\mathrm{e}}$. These points $\lambda_{\mathrm{n}}$ are eigenvalues of $H$. Since the essential spectrum of $H$ is unbounded, and the multiplicity of each $\lambda_{n}$ is one, it follows that there exists an unbounded set $\left\{\beta_{j}\right\}$ of cluster points of $\left\{\lambda_{n}\right\}$. (It should be remarked that the $\lambda_{n}$ are not necessarily arranged in increasing order.)

Let $\Psi_{n}$ be the normalized eigenfunction corresponding to the eigenvalue $\lambda_{n}$. If $c_{n}=\left[e, \Psi_{n}\right]$, then $\sigma_{e}\left(\lambda_{n}\right)=\left|c_{n}\right|^{2}$. But $F_{\lambda_{n}, e}=\alpha_{n} \Psi_{n}$ for some complex number $\alpha_{n}$. Hence

$$
\begin{gathered}
\mathrm{U}_{\mathrm{e}}(\mathrm{e})\left(\lambda_{\mathrm{n}}\right)=\left[\mathrm{e}, \mathrm{F}_{\lambda_{\mathrm{n}}}, \mathrm{e}=1=\bar{\alpha}_{\mathrm{n}} \mathrm{c}_{\mathrm{n}} ; \text { thus } \bar{\alpha}_{\mathrm{n}}=1 / \mathrm{c}_{\mathrm{n}}\right. \text {. Thus } \\
\left\|\mathrm{F}_{\lambda_{\mathrm{n}}, \mathrm{e}}\right\|_{\mathrm{Z}_{\omega}}=\left\|\omega \mathrm{F}_{\lambda_{\mathrm{n}},}, \mathrm{e}_{2}=\right\| \omega \Psi_{\mathrm{n}} \|_{2} /\left|\mathrm{c}_{\mathrm{n}}\right| ; \text { then } \mu_{\mathrm{e}}\left(\lambda_{\mathrm{n}}\right)=\left\|\mathrm{F}_{\lambda_{\mathrm{n}}},\right\|_{\mathrm{Z}_{\omega}}^{2} \sigma_{\mathrm{e}}\left(\lambda_{\mathrm{n}}\right)=\left\|\omega \Psi_{\mathrm{n}}\right\|_{2}^{2}
\end{gathered}
$$

Let $\beta_{j}$ be a cluster point of $\left\{\lambda_{n}\right\}$. Let $\left\{\lambda_{r}\right\}$ converge to $\beta_{j} .\left[\Psi_{\lambda_{r}}, \Psi_{\lambda_{s}}\right]=0$ for $\mathrm{r} \neq \mathrm{s}$. But on any compact interval $[0, M]$, if $N([0, M])$ is the characteristic function of $[1, M]$, then Ascoli's theorem guarantees that $\left\{\mathfrak{N}([0, M]) \Psi_{\lambda_{\mathbf{r}}}\right\}$ has a cluster point $\mathrm{g}_{\mathbf{M}}$ in $\mathrm{L}_{2}$. But if $\gamma_{\mathbf{r}}=\left[\mathrm{g}_{\mathbf{M}}, \mathcal{N}([0, \mathrm{M}]) \Psi_{\lambda_{\mathrm{r}}}\right]$, then the sequence $\gamma_{\mathbf{r}}$ is square summable and $\gamma_{\mathbf{r}}-\left\|\mathcal{N}([0, M]) \Psi_{\lambda_{r}}\right\|^{2}$ converges to 0 . But since $\Psi_{\lambda_{I}}$ is normalized in $\mathrm{L}_{2}$, and $\tau \Psi_{\lambda_{\mathrm{r}}}=\lambda_{\mathrm{I}} \Psi_{\lambda_{\mathrm{r}}}$, we see since the sequence $\lambda_{\mathbf{r}}$ is bounded that $\left\|\Psi_{\lambda_{\mathbf{r}}}\right\|_{\infty}$ is also bounded. It follows that for each $\beta_{j}$, there exists at least one (actually infinitely many) $\Psi_{\lambda_{r(j)}}$ such that $\left\|\omega \Psi_{\lambda_{r(j)}}\right\|_{2} \leq 1 / j^{2}$, and such that $\left|\lambda_{r(j)}-\beta_{j}\right|<1$. It follows that the sequence $\lambda_{r(j)}$ approaches infinity, and that if $\Delta=\left\{\lambda_{r(j)}\right\}, \mu_{e}(\Delta)$ is finite but $\sigma_{\mathrm{e}}(\Delta \backslash[-\mathrm{N}, \mathrm{N}])>0$ for all $\mathrm{N}$. The theorem is proved.

\section{DIAGONALIZATION IN $\mathrm{L}_{2}(\mathrm{X}, \rho)$}

In this section we discuss diagonalization of $P(\Delta)$, not just $P(\Delta) P\left(S_{e}\right)$, and show the equivalence of compactness of $\mathrm{P}(\Delta)$ in $\mathrm{B}\left(\mathrm{Y}, \mathrm{L}_{2}\right)$ and diagonalization in $\mathrm{B}(\mathrm{Y}, \mathrm{Z})$ discussed in the introduction. For situations where the embedding of $\mathrm{Y}$ into $\mathrm{L}_{2}$ is not compact, these properties generally hold for some but not all $\Delta$. In applications to partial differential equations, it is often true that $\mathrm{P}(\Delta)$ is known by other means to be compact for all bounded $\Delta$; an example is the situation of Theorem 5.5. When $\Delta$ is unbounded but the embedding from $Y$ into $Z$ is not compact, compactness and diagonalization become delicate properties of $\Delta$ as we see for an example by the results of section 4 .

Remark: The difference between the following definition and Definition 3.2 is only that we diagonalize $\mathrm{P}(\Delta) \mathrm{r}(\mathrm{H})$, not just $\mathrm{P}(\Delta) \mathrm{P}\left(\mathrm{S}_{\mathrm{e}}\right) \mathrm{r}(\mathrm{H})$. For completeness, however, the definition is given in its entirety. 
Definition 5.1. Assume the hypotheses of Assumptions 1.2 and 3.1 and Estimate 1.3. Let $r$ be a bounded continuous function from the spectrum of $H$ into the complexes. Let $\Delta$ be any Borel subset of $\mathbb{R}$. We say that $Q=P(\Delta) r(H)$ is diagonalizable in $B(Y, Z)$ with respect to $H$ if $B^{\prime} Q g \in L_{2}(X, \rho)$ for all $g \in Y$, and the following is true:

a) for every $\epsilon>0$ there exists a positive integer $\mathbf{k}$ and a finite disjoint family $\left\{\Delta_{\mathrm{i}}\right\}_{\mathrm{i}=1}^{\mathbf{k}}$ of subsets of $\Delta$ such that there exists a finite set $\left\{e_{j}\right\}_{j=1}^{m}$ of orthonormal elements of the domain of $H^{N}$ and a finite set of real numbers $\left\{\lambda_{i, j}\right\}_{i=1}^{k}$ such that $\lambda_{i, j} \in \Delta_{i}$ and such that, injecting $Q \theta$ canonically into $W^{\prime},\left\|\left\{Q-\Sigma_{i=1}^{k} \Sigma_{j=1}^{m} \mu_{e_{j}}\left(\Delta_{i}\right) \bar{r}\left(\lambda_{i, j}\right) R_{\lambda_{i, j}, e_{j}}\right\}(\theta)\right\|_{Z} \leq \epsilon\|\theta\|_{Y}$ for all $\theta \in Y$, where

b) $\lambda_{i, j}$ is in the complement of the exceptional set for $e_{j}$ of Theorem 1.8, so that in particular $F_{\lambda_{i, j} j_{j}} e_{j}$ is as in Theorem 1.8 and $B^{\prime} F_{\lambda_{i, j} j_{j}}, L_{2}(X, \rho)$, and where

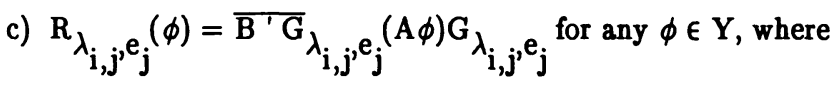

$G_{\lambda_{i, j} j_{j}, e_{j}}=F_{\lambda_{i, j}, e_{j}} /\left\|B^{\prime} F_{\lambda_{i}}, e\right\|_{2}$, and where ${\bar{B}{ }^{\prime} G}_{\lambda, e}$ denotes the complex conjugate of the functional $B^{\prime} G_{\lambda, e}$.

Let $M$ be a family of bounded continuous functions from the spectrum of the restriction of $H$ to $S_{e}$ into $C$. Let $\Delta$ be a Borel subset of $\mathbb{R}$. Let $Q_{M, \Delta}=\{P(\Delta) r(H): r \in M\}$. We say that $\mathrm{Q}_{\mathrm{M}, \Delta}$ is simultaneously diagonalizable in $\mathrm{B}(\mathrm{Y}, \mathrm{Z})$ with respect to $\mathrm{H}$ if for every $\epsilon>0$ there exists $\Delta_{i}, \lambda_{i, j}$ and $e_{j}$ as above, which are independent of $r$, such that

$$
\left\|\left\{Q-\Sigma_{i=1}^{k} \Sigma_{j=1}^{m} \mu_{e_{j}}\left(\Delta_{i}\right) \bar{r}\left(\lambda_{i, j}\right) R_{\lambda_{i, j}, e_{j}}\right\}(\theta)\right\|_{Z} \leq \epsilon\|\theta\|_{Y}
$$

for all $\theta \in \mathrm{Y}$ and all $\mathrm{Q} \in \mathrm{Q}_{\mathbf{M}, \delta}$.

Remark: The implication that diagonalizability implies compactness in $B(Y, Z)$ follows from the fact that any operator with finite dimensional range is compact, and that the compact operators are a closed subset of $\mathrm{B}(\mathrm{Y}, \mathrm{Z})$.

Theorem 5.2. Suppose that the hypotheses of Assumptions $\$ .1$ and 1.2 hold, and that Estimate 1.9 holds. Suppose that $\mathrm{Z}$ contains $\mathrm{L}_{2}(\mathrm{X}, \rho)$, and that the injection from $\mathrm{L}_{2}(\mathrm{X}, \rho)$ into $\mathrm{Z}$ is continuous. Let $\mathrm{E}_{\mathrm{Y}}$ denote the injection from $\mathrm{Y}$ into $\mathrm{L}_{2}(\mathrm{X}, \rho)$, and $\mathrm{E}^{\mathrm{Z}}$ denote the injection from $L_{2}(X, \rho)$ into $Z$. Then

i) $\mathrm{PE}_{\mathrm{Y}}$ is compact in $\mathrm{B}\left(\mathrm{Y}, \mathrm{L}_{2}\right)$ if and only if $\mathrm{P}$ is diagonalizable in $\mathrm{B}(\mathrm{Y}, \mathrm{Z})$ with respect to $\mathrm{H}$, where $\mathrm{P}=\mathrm{P}(\Delta)$ or $\mathrm{P}(\Delta) \mathrm{P}\left(\mathrm{S}_{\mathrm{e}}\right)$; further, $\mathrm{E}^{\mathrm{Z}} \mathrm{PE}_{\mathrm{Y}}$ is compact in $\mathrm{B}(\mathrm{Y}, \mathrm{Z})$ if and only if $\mathrm{PE}_{\mathrm{Y}}$ is compact in $\mathrm{B}\left(\mathrm{Y}, \mathrm{L}_{2}\right)$;

ii) Suppose that $\mathrm{PE}_{\mathrm{Y}}$ is compact in $\mathrm{B}\left(\mathrm{Y}, \mathrm{L}_{2}\right)$. Then for any bounded continuous function $\mathrm{r}$ from the spectrum of $\mathrm{H}$ into the complexes, and any Borel subset of $\mathbb{R}$, including $\mathbb{R}$ itself, $\operatorname{Pr}(\mathrm{H})$ is diagonalizable with respect to $\mathrm{H}$. Furthermore, if $\mathrm{M}$ is a set of continuous functions from the spectrum of $\mathrm{H}$ into $\mathrm{C}$, and $\mathrm{M}$ is uniformly bounded and equicontinuous on $\Delta$, then $\mathrm{Q}_{\mathrm{M}, \Delta}$ is simultaneously diagonalizable. 
Proof: We consider the case $\mathrm{P}=\mathrm{P}(\Delta)$. The other case is done the same way, except that it is easier. Note that $L_{2}(X, \rho)$ is the direct sum of countably many subspaces $S_{e_{i}}$, where $e_{i}$ is in the domain of $H^{N}$, and $\left\|e_{i}\right\|=1$. If $P(\Delta) E_{Y}$ is compact in $B\left(Y, L_{2}\right)$, an elementary argument shows that for every $\epsilon>0$ there exists a positive integer $m$ such that

$$
\left\|\mathrm{P}(\Delta) \mathrm{E}_{\mathrm{Y}}-\mathbf{\Sigma}_{\mathrm{i}=1}^{\mathrm{m}} \mathrm{P}\left(\mathrm{S}_{\mathrm{e}_{\mathrm{i}}}\right) \mathrm{P}(\Delta) \mathrm{E}_{\mathrm{Y}}\right\|_{\mathrm{B}\left(\mathrm{Y}, \mathrm{L}_{2}\right)}<\epsilon .
$$

Also, there exists a tower $\left\{\xi_{j}\right\}$ of Borel measurable subsets of $\mathbb{R}$ such that $\sigma_{e_{i}}\left(\mathbb{R} \mid \cup_{j=1}^{\infty} \xi_{j}\right)=0$ for all $\mathrm{i} \leq \mathrm{m}$, and such that, for $\lambda \in \xi_{\mathrm{j}},\left\|\mathrm{B}^{\prime} \mathrm{F}_{\lambda, \mathrm{e}_{\mathrm{i}}}\right\|_{2} \leq \mathrm{j}$ for all $\mathrm{i} \leq \mathrm{m}$. Let $\nu_{\mathrm{j}}=\xi_{\mathrm{j}} \backslash \xi_{\mathrm{j}-1}$. Let $\Delta_{\mathrm{j}}=\Delta n \nu_{\mathrm{j}}$. Again using the fact that $\mathrm{P}(\Delta) \mathrm{E}_{\mathrm{Y}}$ is compact, we see that for the above $\epsilon$, there exists a $\mathrm{J}$ such that

$$
\left\|\Sigma_{j=1}^{J} P\left(\Delta_{i}\right) \Sigma_{i=1}^{m} P\left(S_{e_{i}}\right) E_{Y}-\Sigma_{i=1}^{m} P\left(S_{e_{i}}\right) P(\Delta) E_{Y}\right\|_{B\left(Y, L_{2}\right)}<\epsilon .
$$

Hence, since $r(H)$ is uniformly bounded by some constant $\Gamma$ in operator norm for $r \in M$, it follows that for all $r \in M$,

$$
\left\|\mathrm{P}(\Delta) \mathrm{r}(\mathrm{H})\left\{\mathrm{E}_{\mathbf{Y}}-\Sigma_{\mathrm{j}=1}^{\mathrm{J}} \mathrm{P}\left(\Delta_{\mathrm{j}}\right) \Sigma_{\mathrm{i}=1}^{\mathrm{m}} \mathrm{P}\left(\mathrm{S}_{\mathrm{e}_{\mathrm{i}}}\right) \mathrm{E}_{\mathrm{Y}}\right\}\right\|_{\mathrm{B}\left(\mathrm{Y}, \mathrm{L}_{2}\right)}<2 \epsilon \Gamma .
$$

The implication that compactness implies diagonalizability follows immediately, upon using the implication ii) $\rightarrow$ iii) of Theorem 3.3 for each $S_{e_{i}}$, for $i \leq m$ together with the fact that the injection from $\mathrm{L}_{2}$ into $\mathrm{Z}$ is bounded.

Suppose that $\mathrm{P}(\Delta) \mathrm{E}_{\mathrm{Y}}$ is not compact. Then there exists a bounded sequence $\mathrm{y}_{\mathbf{n}}$ in $\mathrm{Y}$ such that $P(\Delta) y_{n}$ has no convergent subsequence in $L_{2}$. There exists a weakly convergent subsequence to the sequence $P(\Delta) y_{n}$, which we assume without loss of generality is the original sequence; suppose $P(\Delta) y_{n}$ converges weakly to $g$. If $P(\Delta)$ is diagonalizable in $B(Y, Z)$, then by the remark preceding the theorem $E^{Z} P(\Delta) E_{Y}$ is compact in $B(Y, Z)$; thus $E^{Z} P(\Delta) y_{n}$ has a subsequence, which again we assume is the original sequence, which converges to $\mathrm{E}^{\mathrm{Z}} \mathrm{g}$ in $\mathrm{Z}$ and therefore in $\mathrm{Y}^{\prime}$. Now $\mathrm{E}^{\mathrm{Z}} \mathrm{P}(\Delta) \mathrm{y}_{\mathrm{n}}\left(\mathrm{y}_{\mathrm{n}}\right)$ converges to $\|g\|_{2}^{2}$, by an elementary argument. But $\mathrm{E}^{\mathrm{Z}} \mathrm{P}(\Delta) \mathrm{y}_{\mathrm{n}}\left(\mathrm{y}_{\mathrm{n}}\right)=\left\|\mathrm{P}(\Delta) \mathrm{y}_{\mathrm{n}}\right\|_{2}^{2}$, which then converges to $\|\mathrm{g}\|_{2}^{2}$. By another elementary argument, it follows that $P(\Delta) y_{n}$ converges to $g$ in $L_{2}$, a contradiction. Hence $E^{Z} P(\Delta) E_{Y}$ is not compact, so that $P(\Delta)$ is not diagonalizable in $B(Y, Z)$. The first assertion is proved; because we have proved that diagonalizability implies compactness in $B(Y, Z)$ which implies compactness in $B\left(Y, L_{2}\right)$ which implies diagonalizability.

Remark: We now test the results against the abstract Schrödinger equation, which is more difficult than, for example, the abstract heat equation, because damping is not present.

Corollary 5.3. Suppose that the hypotheses of Theorem 5.2 hold. Suppose that $\mathrm{P}(\Delta) \mathrm{E}_{\mathrm{Y}}$ is a compact operator in $\mathrm{B}(\mathrm{Y}, \mathrm{Z})$. Then for every pair $\mathrm{T}, \epsilon$ of positive real numbers, there exists a finite set $\left\{\mathrm{F}_{\lambda_{\mathrm{i}}}\right\}$ of elements of $\mathrm{Z}$ such that $\mathrm{H}^{\prime} \mathrm{F}_{\lambda_{\mathbf{i}}}=\lambda_{\mathbf{i}} \mathrm{F}_{\lambda_{\mathrm{i}}}$ for some $\lambda_{\mathbf{i}} \in \Delta \cap$ spectrum (H), and such that $\left\|\mathrm{P}(\Delta) \mathrm{e}^{\mathrm{iHt}} \phi-\Sigma \mathrm{e}^{-\mathrm{i} \lambda \mathrm{t}} \overline{\mathrm{F}}_{\lambda_{\mathrm{i}}}(\phi) \mathrm{F}_{\lambda_{\mathrm{i}}}\right\|_{\mathrm{Z}} \leq \epsilon\|\phi\|_{\mathrm{Y}}$ for all $\phi$ in $\mathrm{Y}$, and all $\mathrm{t}$ such that $|\mathrm{t}| \leq \mathrm{T}$. 
Proof: By Theorem 5.2, $\left\{\mathrm{P}(\Delta \mathrm{n}[-\mathrm{n}, \mathrm{n}]) \mathrm{e}^{\mathrm{iHt}}:|\mathrm{t}| \leq \mathrm{T}\right\}$ is simultaneously diagonalizable in $B(Y, Z)$, since $\left\{e^{i \lambda t}: \lambda \in[-n, n],|t| \leq T\right\}$ is equicontinuous. Using the compactness of $E^{Z} P(\Delta) E_{Y}$ again as in the proof of Theorem 5.2, we see that for any $\epsilon>0$ there exists a positive integer $n$ such that $\left\|\mathrm{P}(\Delta n[-n, n]) \mathrm{e}^{\mathrm{iHt}}-\mathrm{P}(\Delta) \mathrm{e}^{\mathrm{i} H \mathrm{t}}\right\|_{\mathrm{B}(\mathrm{Y}, \mathrm{Z})}<\epsilon$. The result follows from Theorem 5.2.

Example: In Example 2.3, let

$$
\begin{gathered}
\mathrm{Y}=\left\{\theta \in \mathrm{L}_{2}\left(\mathbb{R}^{\mathrm{n}}\right) \mid\left(|\mathrm{x}|^{2}+1\right)^{\gamma}\left(\Sigma_{\mathrm{i}=1}^{\mathrm{n}} \partial^{2} / \partial \mathrm{x}_{\mathrm{i}}^{2}-1\right)^{\mathrm{M}_{\theta \in \mathrm{L}_{2}}\left(\mathbb{R}^{\mathrm{n}}\right), \text { and }}\right. \\
\|\theta\|_{\mathrm{Y}}=\|\left(|\mathrm{x}|^{2}+1\right)^{\gamma}\left(\Sigma_{\mathrm{i}=1}^{\mathrm{n}} \partial^{2} / \partial \mathrm{x}_{\mathrm{i}}^{2}-1\right)^{\mathbf{M}_{\theta \|_{2}}}
\end{gathered}
$$

This example yields the next corollary.

Remark: In the next result, we look for situations where the approximation property holds for the whole operator $\mathrm{e}^{\mathrm{iHt}}$, so that we may take $\Delta=\mathbb{R}$. We find that this is possible in great generality, but at the price of substantially restricting the space $Y$ and increasing the space $Z$, which has the practical effect of substantially weakening the error estimate from, say, the situation of Section 4.

Corollary 5.4. Suppose that $\tau$ is a partial differential expression on $\mathbb{R}^{\mathbf{n}}$ such that the coefficients of $\tau$ are infinitely differentiable, and such that any derivative of a coefficient has at most polynomial growth at infinity. Suppose $\mathrm{H}$ is any self-adjoint operator in $\mathrm{L}_{2}\left(\mathbb{R}^{\mathrm{n}}\right)$ such that the rapidly decreasing functions are contained in the domain of $\mathrm{H}$, and such that $\mathrm{H} \phi=\tau \phi$ for all $\phi$ in the domain of $\mathrm{H}$, where the derivatives are taken in the distribution sense. Let $\gamma>\mathrm{n} / 4$. Then for any positive real numbers $\mathrm{T}, \epsilon$ there exists a finite set $\left\{\Psi_{\lambda_{\mathrm{i}}}\right\}$ of tempered distributions such that ${ }^{\prime} \Psi_{\lambda_{i}}=\lambda_{i} \Psi_{\lambda_{i}}$ for some $\lambda_{i} \in$ spectrum $(\mathrm{H})$, and such that $\left\|\mathrm{e}^{\mathrm{i} H \mathrm{t}} \theta-\Sigma \mathrm{e}^{\mathrm{i} \lambda \mathrm{t}} \mathrm{c}_{\lambda_{\mathrm{i}}}(\theta) \Psi_{\lambda_{\mathrm{i}}}\right\|_{\mathrm{Z}} \leq \epsilon\|\theta\|_{\mathrm{Y}}$ for all $\theta \in \mathrm{Y}$ and $|\mathrm{t}| \leq \mathrm{T}$, where

i) $\|\mathrm{F}\|_{\mathrm{Z}}=\left\|\left(|\mathrm{x}|^{2}+1\right)^{-\gamma}\left(\Sigma_{\mathrm{i}=1}^{\mathrm{n}} \partial^{2} / \partial \mathrm{x}_{\mathrm{i}}^{2}-1\right)^{-\mathrm{M}} \mathrm{F}\right\|_{2}$ and $\mathrm{Z}$ consists of all tempered distributions $\mathrm{F}$ such that this norm is finite;

ii) $\Psi_{\lambda_{i}} \in \mathrm{Z}$;

iii) $\mathrm{M}>\mathrm{n} / 4$;

iv) $\|\theta\|_{\mathrm{Y}}=\left\|\left(|\mathbf{x}|^{2}+1\right)^{\gamma}\left(\Sigma_{\mathrm{i}=1}^{\mathrm{n}} \partial^{2} / \partial \mathrm{x}_{\mathrm{i}}^{2}-1\right)^{\mathrm{M}} \theta\right\|_{2}$, and $\mathrm{Y}$ is the set of all elements of $\mathrm{L}_{2}\left(\mathbb{R}^{\mathrm{n}}\right)$ such that this norm is finite; and

v) $\mathrm{c}_{\lambda_{\mathrm{i}}}(\theta)=\int \theta \overline{\mathrm{F}}_{\lambda_{\mathrm{i}}} \mathrm{d} \rho$

Remark: The complex conjugates have disappeared from the approximation formula because here we do not need to consider $\mathrm{Z}$ as a subset of $\mathrm{Y}^{\prime}$.

Proof: It is not difficult to see that the restriction of $B^{\prime}$ to $L_{2}\left(\mathbb{R}^{n}\right)$ is a compact operator. Call this operator $\mathrm{V}$. By taking adjoints, we see that $\mathrm{V}^{*}$ is also compact. But $\|\theta\|_{\mathrm{Y}}=\left\|\left(\mathrm{V}^{*}\right)^{-1} \theta\right\|_{2} ;$ the compactness of the injection from $\mathrm{Y}$ into $\mathrm{L}_{2}\left(\mathbb{R}^{\mathrm{n}}\right)$ follows immediately. Assumption 3.1 holds automatically. The corollary is now a consequence of Corollary 5.3. 
Theorem 5.5. Let $\mathrm{H}$ be the Friedrichs extension in $\mathrm{L}_{2}(\Omega)$ of the restriction of $\tau$ to $\mathrm{C}_{0}^{\infty}(\Omega)$, where $\Omega$ is an open subset of $\mathbb{R}^{n}$, and where $\tau$ is a partial differential expression with $\mathrm{C}^{\infty}$ coefficients on $\Omega$, such that $[\tau \phi, \phi] \geq-\mathrm{K}[\phi, \phi]$ for all $\phi \in \mathrm{C}_{0}^{\infty}(\Omega)$, where $\mathrm{K}$ is a positive constant. Let $\omega$ be any positive, bounded, $\mathrm{C}^{\Phi}$ element of $L_{2}(\Omega)$; let $\mathrm{Y}_{\omega}=\left\{\mathrm{f}: \mathrm{f} / \omega \in \mathrm{L}_{2}(\Omega)\right.$; let $\mathrm{Z}_{\omega}=\left\{\mathrm{f}: \omega \mathrm{f} \in \mathrm{L}_{2}(\Omega)\right\}$; equip these spaces with the obvious norms. Suppose that for some $\mathrm{N}$, the domain of $\mathrm{H}^{\mathrm{N}}$ is contained in $\mathrm{L}_{\infty}(\Omega)$. Then for any bounded set $\Delta$, and any continuous function $\mathrm{r}$ from the spectrum of $\mathrm{H}$ into the complexes, $\mathrm{P}(\Delta) \mathrm{r}(\mathrm{H})$ is diagonalizable in $\mathrm{B}\left(\mathrm{Y}_{\omega}, \mathrm{Z}_{\omega}\right)$.

Proof: Since the range of $P(\Delta)$ is contained in the domain of all powers of $H$, it follows in particular that it is contained in $\mathrm{L}_{\infty}(\Omega)$. It was shown Assertion $\mathrm{i}$ ) of Theorem 5.2 that $\mathrm{E}^{\mathrm{Z}} \mathrm{P}(\Delta) \mathrm{E}_{\mathrm{Y}}$ is compact in $\mathrm{B}(\mathrm{Y}, \mathrm{Z})$ if and only if $\mathrm{P}(\Delta) \mathrm{E}_{\mathrm{Y}}$ is compact in $\mathrm{B}\left(\mathrm{Y}, \mathrm{L}_{2}\right)$, where $\mathrm{Y}=\mathrm{Y}_{\omega}$ and $\mathrm{Z}=\mathrm{Z}_{\omega^{\prime}}$. But by using Lemma 1.4, it is easy to see that the mapping $\mathrm{E}^{\mathrm{Z}} \mathrm{P}(\Delta)$ is Hilbert-Schmidt, and therefore compact, for bounded $\Delta$. Hence $\mathrm{E}^{\mathrm{Z}} \mathrm{P}(\Delta) \mathrm{E}_{\mathrm{Y}}$ is compact, and the theorem now follows from Theorem $\mathbf{5 . 2}$.

ACKNOWLEDGMENT. The author was supported by British SERC grant number GR/E41924 at the University of Wales, Cardiff, April-July 1988, when this paper was begun.

\section{REFERENCES}

1. J. Berezanskii, Eigenfunction Expansions, Providence: American Mathematical Society, 1968.

2. N. Dunford and J. T. Schwartz, Linear operators, vol. 2, New York: Wiley-Interscience, 1963.

3. R. E. Edwards, Functional Analysis, Theory and Applications, New York: Holt, Rinehart, and Winston 1965.

4. D. B. Hinton and R. M. Kauffman, Finite Eigenfunction Approximations for Second Order Ordinary Differential Operators with continuous spectrum, preprint (1991).

5. T. Poerschke, G. Stolz, and J. Weidmann, Expansions in Generalized Eigenfunctions of Selfadjoint Operators, Math. Z. 202, (1989) 397-408.

6. T. Poerschke and G. Stolz, On Eigenfunction Expansions and Scattering Theory, Preprint (1990).

7. A. P. Robertson and W. J. Robertson, Topological Vector Spaces, second edition, Cambridge Tracts in Mathematics 53, Cambridge: Cambridge University Press, 1973.

8. B. Simon, Schrödinger Semigroups, Bull. Am. Math. Soc. (New Series) 7, (1982) 447-526.

9. J. Weidmann, Linear Operators in Hilbert Spaces, Berlin Heidelberg New York: Springer-Verlag, 1980. 


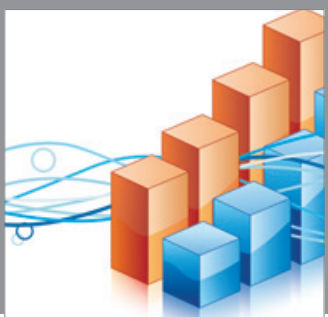

Advances in

Operations Research

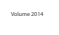

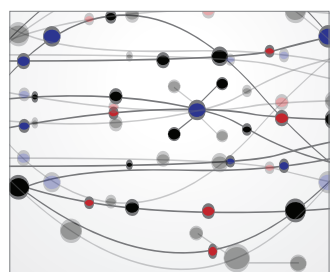

\section{The Scientific} World Journal
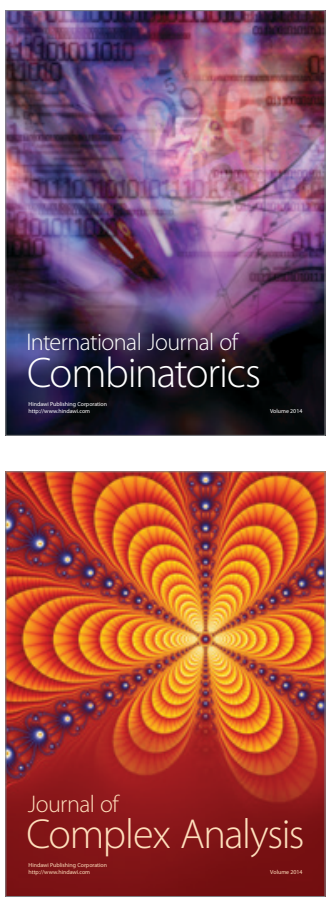

International Journal of

Mathematics and

Mathematical

Sciences
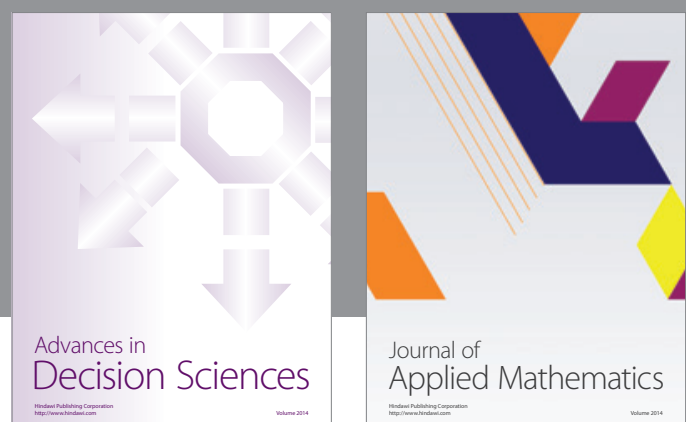

Journal of

Applied Mathematics
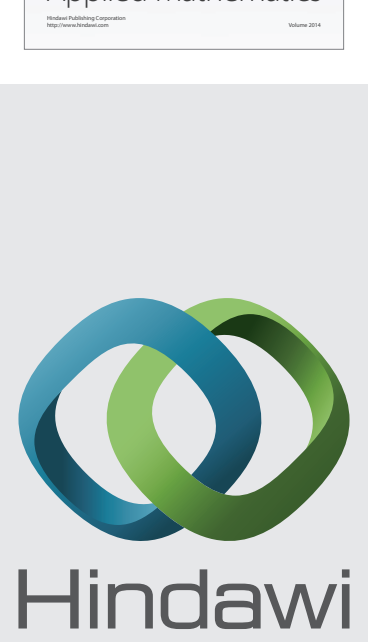

Submit your manuscripts at http://www.hindawi.com
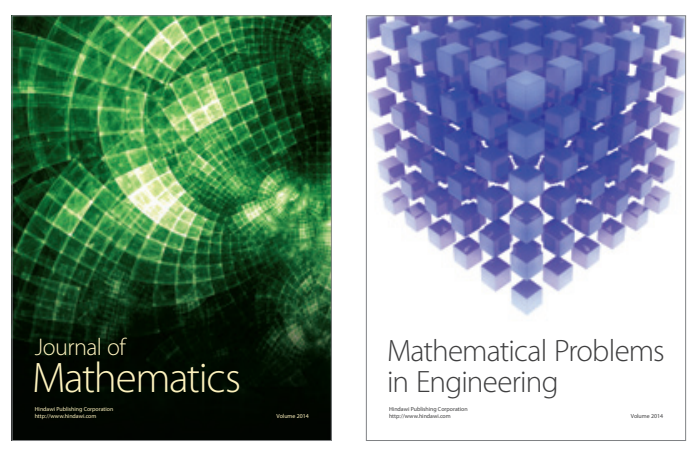

Mathematical Problems in Engineering
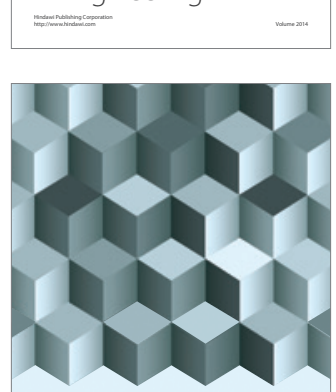

Journal of

Function Spaces
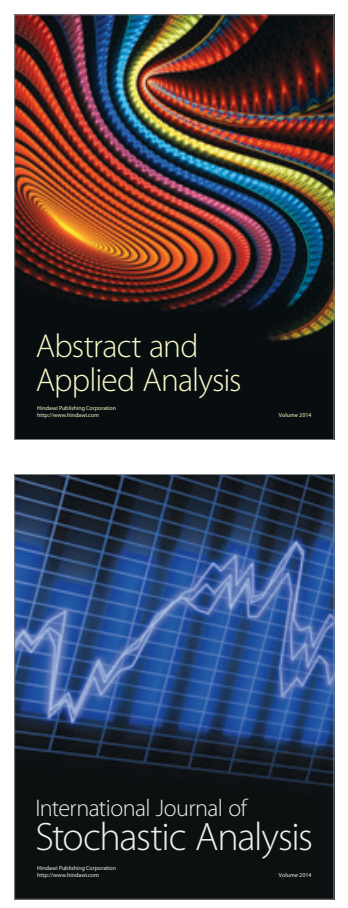

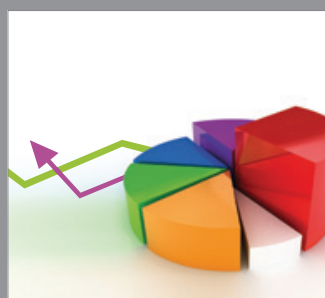

ournal of

Probability and Statistics

Promensencen
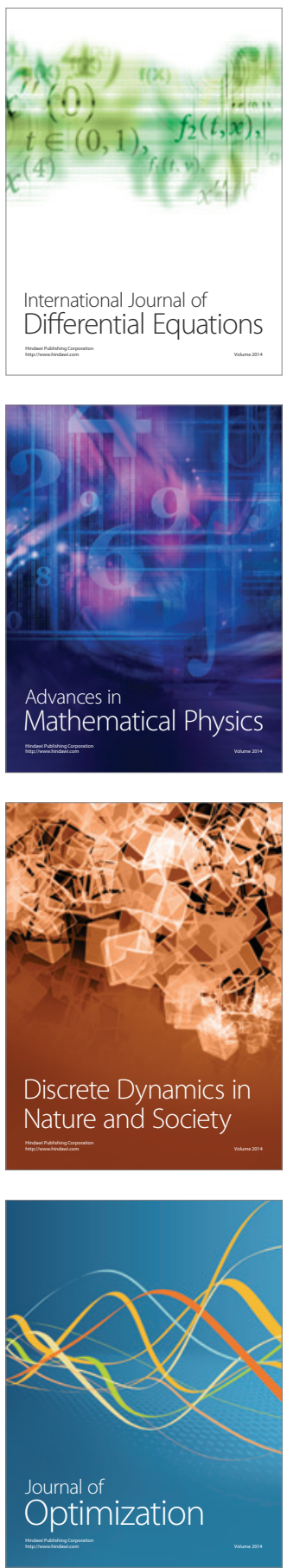